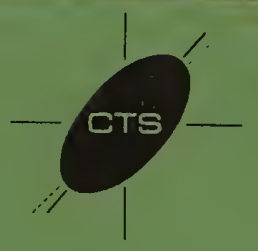

Collaborative Testing Services, Inc.

\title{
COLLABORATIVE REFERENCE PROGRAM FOR RUBBER
}

ANALYSES NO. 40 APRIL - JUNE 1979

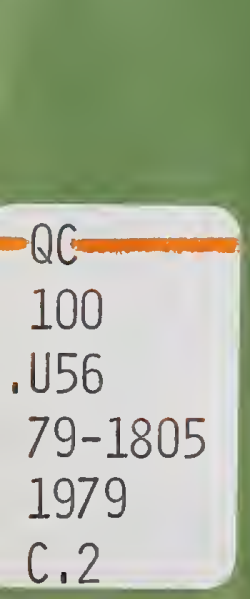

U.S. DEPARTMENT OF COMMERCE National Bureau of Standards 


\section{TAPPI Paper and Board ( 6 times per year)}

Bursting strength

Tearing strength

Tensile breaking strength

Elongation to break

Tensile energy absorption

Folding endurance

Stiffness

Air resistance

Grammage

\author{
Smoothness \\ Surface pick strength \\ $K \& N$ ink absorption \\ $\mathrm{pH}$ \\ Opacity \\ Blue reflectance (brightness) \\ Specular gloss, $75^{\circ}$ \\ Thickness \\ Concora (flat crush) \\ Ring crush
}

FKBG-API Cortainerboard (48 times per year)

Mullen burst of linerboard

Concora test of medium

MCCA Color and Appearance (4 times per year)

Gloss at $60^{\circ}$

Color and color difference

CTS Rubber (4 times per year)

Tensile strength, ultimate el.ongation and tensile stress

Hardness

Mooney viscosity

Vulcanization properties

CTS Thermal Insulation Materials (2 times per year)

19 test methods for thermal insulation materials covering: thermal properties; strength properties; dimensions, stability, and density properties; fire properties; and properties of vapor barriers

ASTM Cement ( 2 times per year)

Chemical (11 chemical components)

Physical ( 8 characteristics)

AASHTO Bituminous

Asphalt cement ( 2 times per year)

Cutbacks (once a year)

NBS Collaborative Reference Programs A05 Technology Building National Bureau of Standards Washington, DC 20234 


\title{
INTERLABORATORY PROGRAMS FOR RUBBER
}

\author{
Analyses No. 40 \\ April - June 1979
}

\author{
J. Horlick
}

Office of Testing Laboratory Evaluation Technology

Office of Engineering Standards

National Engineering Laboratory

T. L. Cummings, Research Associate, Collaborative Testing Services, Inc.

G. W. Bullman

Polymers Science and Standards Division

Center for Materials Research

National Measurement Laboratory

\section{U. S. DEPARTMENT OF COMMERCE}

National Bureau of Standards 


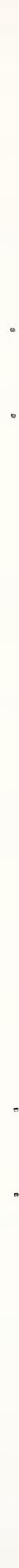


This report summarizes the test results for the second quarter of 1979. The tests cover the four areas in the NBS Collaborative Reference Programs for Rubber: Tensile Properties, Hardness, Mooney Viscosity, and Vulcanization Properties.

For each of the four areas, there is a set of summary tables followed by a table of data and analysis by laboratory and a graphical presentation of the data and analysis. Where applicable, the tables of data have the English and Metric expressions side-by-side. Additional details are given in the section "Key to Tables and Graphs."

If there are questions or comments on the notes, the analyses, or the reports in general, contact Jeffrey Horlick at (301) 921-2946.

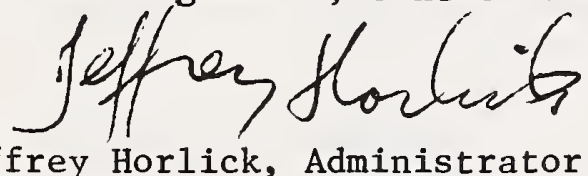

NBS Collaborative Reference Programs

Office of Testing Laboratory Evaluation Technology

August 9, 1979 


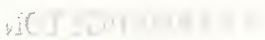

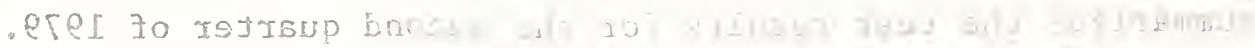

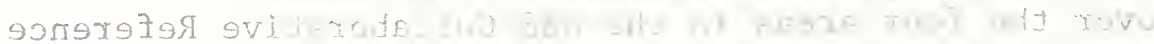

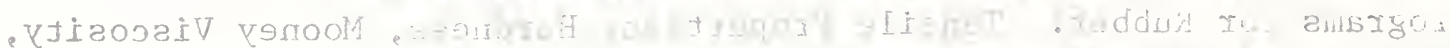

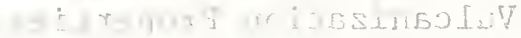

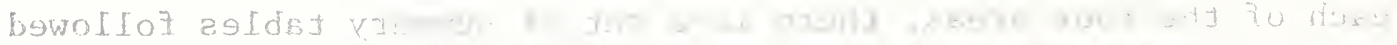

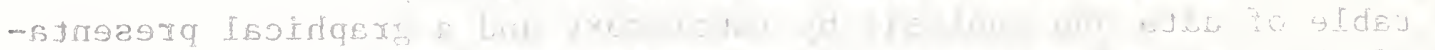

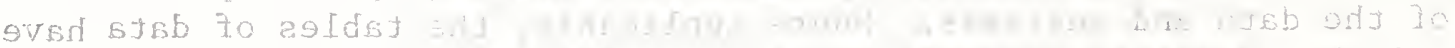

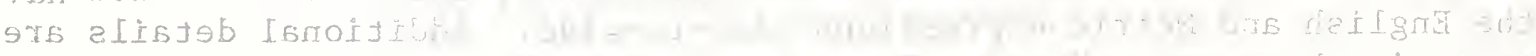

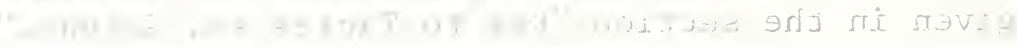

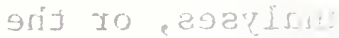
$.9+\operatorname{es}-1:$ 
TABLE OF CONTENTS

$\underline{\text { Page }}$

Introduction

Table of Contents 1

Key to Tables and Graphs 2

Program 1: Tensile strength, ultimate elongation and stress at $300 \%$ elongation 5

$\begin{array}{ll}\text { Program 2: Hardness } & 13\end{array}$

$\begin{array}{ll}\text { Program 4: } & 17\end{array}$

Program 5: Vulcanization characteristics determined with oscillating-disk cure meter 
LAB CODE Confidential laboratory identification number known only to the participant and the Collaborative Reference Program staff.

F

$\mathrm{X}$

*

MEAN

$\%$ DEV

REL SDR

VAR CODE

GR MEAN

SD MEANS
A flag identifying results that are extreme in comparison with the other results.

- The plotted point for the indicated laboratory lies outside of the $99 \%$ error ellipse (not shown); ie, assuming normal distribution, $99 \%$ of laboratories similar to those participating in the program will be represented by points lying within the $99 \%$ ellipse.

- The plotted point for the indicated laboratory lies outside of the $95 \%$ error ellipse shown on graphs, but inside the $99 \%$ ellipse.

The arithmetic average of the two median values for the two sheets or samples of the same material.

The deviation or difference of the laboratory MEAN from the GR. MEAN (see below), expressed as a percent of the GR. MEAN .

The ratio of the SDR (standard deviation of replicate measurements within a laboratory) to the AVER SDR (see below). Extreme values, ie, values that are likely to occur by chance less than one time in a hundred as determined by the chisquare test, are marked with an "X".

A code number designating a particular test instrument, set of environmental conditons, procedure, unit used, or other variation. The code " 01 " designates the instrument, conditions and procedure specified at the top of the page either explicitly or in the cited ASTM Standard, and the unit of test shown at the top of the first column of data. A ' + ' in front of the VAR CODE indicates that the data has been excluded from the grand means due to a non-standard variation of the possibilities mentioned above, or the data is extreme.

The arithmetic average (grand mean) of all the laboratory MEAN values, excluding those flagged (F) with an " $\mathrm{X}$ ".

The standard deviation among the laboratory MEAN values included in the GR. MEAN. 
AVER SDR The arithmetic average of all the standard deviations of within laboratory replication, excluding those excluded from the GR. MEAN and excluding any additional ones for which the REL SDR has been flagged.

\section{GRAPH}

For each laboratory the MEAN for the second material is plotted against the MEAN for the first material, with each point representing a laboratory. The horizontal and vertical lines are the GR. MEAN values. The dashed line is drawn at $45^{\circ}$. The solid sloping line, which may or may not lie close to the $45^{\circ}$ line, is the major axis of the ellipse. The ellipse is drawn so that, on the average, it will include $95 \%$ of the points representing the laboratories. The plotted symbols $\mathrm{X}$ and * used to represent results falling outside the ellipse are explained under "F" above. Laboratories inside the ellipse (no flag in the F column) are plotted as an 0 .

The graph is plotted with an ellipse when there are 20 or more laboratories in the analysis. When there are 10 through 19 laboratories in the analysis, the graph is plotted but the ellipse is omitted. When there are fewer than 10 laboratories retained in the Grand Mean the graph is not plotted.

For development of the theory, see the paper by J. Mandel and T.W. Lashof, Interpretation and Generalization of Youden's Two-Sample Diagram, J. of Quality Technology, Vol. 6, pp 22-36, Jan. 1974.

\section{SUMMARY OF ANALYSES}

LABS INCL Number of laboratories included in the GR. MEANs •

LABS OMIT Number of laboratories reporting data but excluded from the GR. MEANs.

\section{STANDARD DEVIATIONS}

LABS Same as the SD MEANs (see above)

SHEETS Standard deviation between the two sheets or samples of the same material.

REPL Same as AVER SDR (see above)

\section{PRECISION OF METHODS}

REPL CRP The number of replicate measurements per sheet or sample, as specified in the Collaborative Reference Program.

REPL ASTM The number of replicate measurements specified for a test result in the designated ASTM Standard. 
REPEAT The repeatability, a measure of the within laboratory precision, i.e., of the ability of the test technician to repeat his test result: two test results obtained by the same technician on the same homogeneous sample of material may be expected $95 \%$ of the time to agree within the repeatability.

REPROD The reproducibility, a measure of the between laboratory precision: two test results obtained in different laboratories may be expected $95 \%$ of the time to agree within the reproducibility.

ABSOLUTE Values of REPEAT and REPROD expressed in the units of measurement.

PERCENT Values of REPEAT and REPROD expressed as a percent of the GR. MEANs. 
TENSILE STRENGTH, ULTIMATE ELONGATION, AND STRESS AT 300\% ELONGATION

$$
\text { NOTES }
$$

Materials B91 and B92 were sheets of the same vulcanized rubber.

Similarly, materials B93 and B94 were alike.

V100 results were obtained at NBS using a pendulum tester.

All participants used Die C in ASTM D 412 with the following exceptions:

V126 used Die 2 in BS903

V0146 and V208 did not specify a Die

V225 used ASTM Die D

INSTRUMENTS

Instrument

Electronic Manual

Electronic Automatic

Pendulum Manual

Pendulum Automatic
Number of Labs Percent

\begin{tabular}{rr}
21 & $36 \%$ \\
20 & $35 \%$ \\
15 & $26 \%$ \\
2 & $3 \%$ \\
\hline 58 & $100 \%$
\end{tabular}

RELATIVE HUMIDITY

Relative Number Humidity of Labs Percent

\begin{tabular}{lll} 
Below $45 \%$ & 14 & $24 \%$ \\
Above 55\% & 14 & $24 \%$ \\
$45 \%-55 \%$ & 19 & $33 \%$ \\
Not Specified & 11 & $19 \%$ \\
\cline { 2 - 3 } & $\frac{100 \%}{58}$ &
\end{tabular}

SUMMART GF ANALYSES

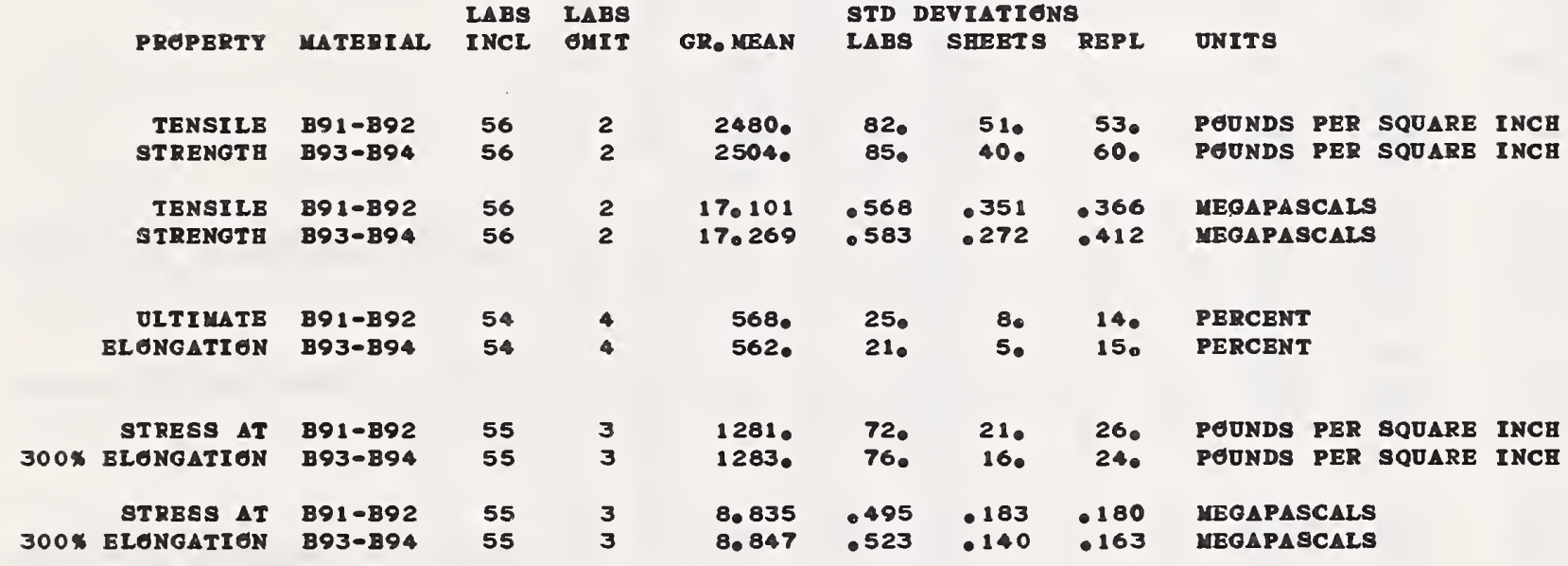

PRECISION OP METHEDS

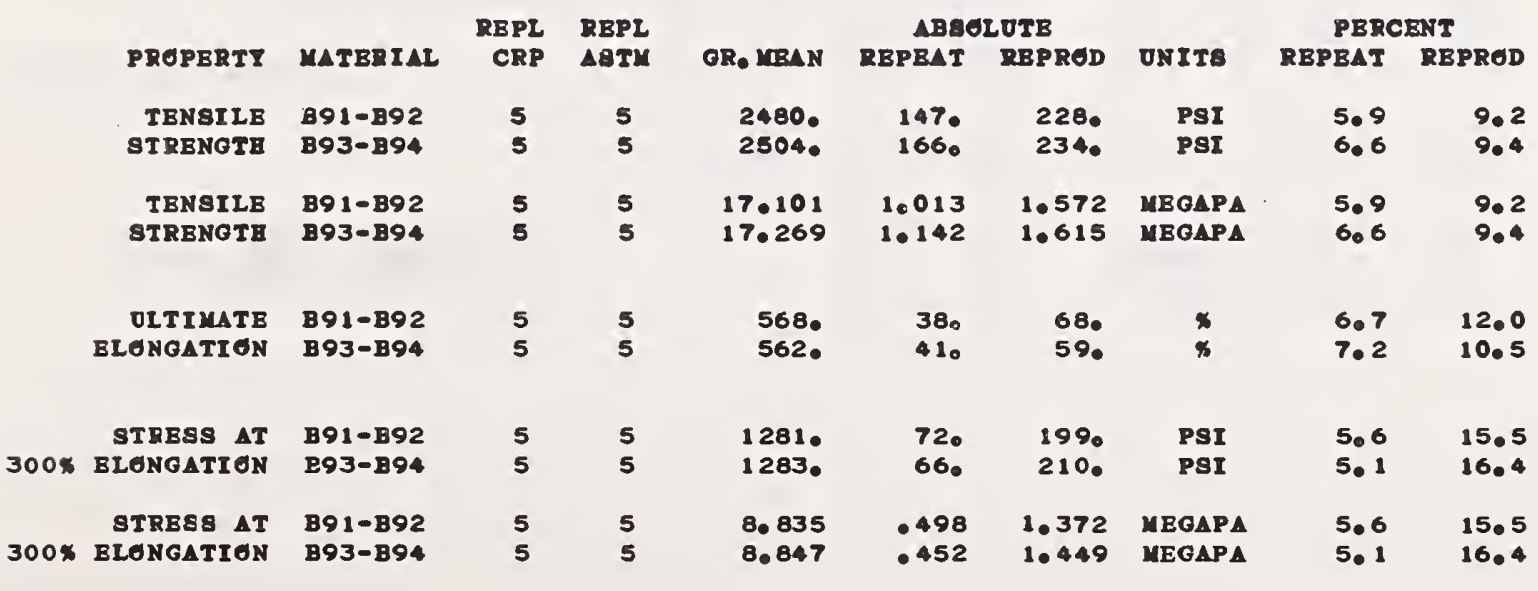


INTEFLABORATORY PROGRAM ON BVALUATIGN GF RUBEER

TENSILE STRENGTB - POUNDS PER SQUARE INCH

APRIL 1979

MATERTAT B93-B94

MATERIAL B91-B92 CEMMERCIAL TIRE TREAD MEAN MEAN \% REL

$\begin{array}{llll}\text { LABDE F PSI MEGAPA DEV SDR } & \end{array}$

v0062

v0063

v0067

vo069

$\begin{array}{rrrr}2297 . & 15.845 & -7.3 & .84 \\ 2504 . & 17.272 & 1.0 & 089 \\ 2417 . & 16.672 & -2.5 & .49\end{array}$

2417. $16.672=2.5 \quad .49$

2520. $17.375 \quad 1.61 .50$

2442 . $16,845-1.51 .06$

vo072

vo073

vo076

v0078

v0083

voo 84

v0085

v0087

v0088

vo092

vo095

vo 100

v0 102

v0111

vo117

vo 123

vo 126

vo1 28

vo141

vo144

vo 144B

vo 146

vo149

vo150

vo154

vo 156

vo 158

vo 160

vo 164

vo 166

v0168

v0169

vo 176

vo 184

vo 190

vo 199

v0 207

v0208

V0213

vo 214

vo 220

v0223

v0224

v0225

vo 232

vo 233

v0235

vo238

vo 243

vo2 44

V0 $245 A$

v0245B

vo 250

\section{0。 18.414 7.7 2.28X}

$\begin{array}{llll}2512 . & 17.328 & 103 & .99\end{array}$

$25140 \quad 17.338 \quad 1.4 \quad .58$

2425. $16.724 \quad-2.2 \quad .97$

2545. $17.552 \quad 2.6 \quad 1.57$

2444. $16.854 \quad-1.4 \quad 1.13$

2450. $16.900-1.21 .73$

2323. $16.021 \quad-6.3 \quad 2,28 \mathrm{x}$

$24000 \quad 16.552 \quad-3.2 \quad 1.64$

2500. $17.241 \quad \bullet \quad 1.37$

2560. $17.655 \quad 3.2 \quad .98$

2320. $16.000 \quad-6.4 \quad 1.11$

$2515 . \quad 17.345 \quad 1.4 \quad 81$

2437. $16.810 \quad-1.7 \quad .63$

2435. $16.793-1.8 \quad .73$

2596. 17.905 $4.7 \quad 1.40$

2510. 17.310 1.2 2.30X

2584. $17.824 \quad 4.2 \quad .63$

2570. $17.724 \quad 3.6 \quad .55$

2525. 17.414 $1.8 \quad .79$

2437. $16.810 \quad-1.7 \quad .97$

2490. 17.172 .4 1.42

2437. $16.810-1.7 \quad .50$

2530. $17.448 \quad 2.0 \quad 1.12$

24750 $17.069 \quad-.2 \quad 1.21$

2495. 17.204 $06 \quad .63$

$\begin{array}{llll}2422 . & 16.704 & -2.3 & 053\end{array}$

2440. $16.831-1.6 \quad 1.68$

2595. 17.897 $4.7 \quad 1.81$

2437. $16.804-1.7 \quad .85$

2495. $17.207 \quad .6 \quad .55$

2420. $16.690 \quad-2.4 \quad .67$

2522

$16.828 \quad-1.6 \quad 1.05$

2570. $17.724 \quad 3.6 \quad .65$

2545。 $17.555 \quad 2.7 \quad .82$

2415. $16.654 \quad-2.6 \quad .65$

2587. 17.845 4.3 1.27

2500. 17.241.8.99

2575. $17.759 \quad 3.8 \quad 1.08$

2436. 16.802 - $1.8 \quad 1.06$

2392. $16.500 \quad-3.5 \quad .63$

2447. $16.879-1.3 \quad .68$

2440, $16.831=1.6-74$

2370. $16.345 \quad-4.4 \quad 1.15$

2564. $17.683 \quad 3.4 \quad 1.72$

2589. 17.855 4.4 2. $15 \mathrm{X}$

2334. $16.100 \quad-5.9 \quad 1.21$

2389. $16.479-3.6 \quad .69$

2575. 17.759 3.8 .95

2480. 17.101 - GR. MEAN -

82. 568 - SD MEANS -

53. 360 - 1 VER 8DR -
2503. 17.259 .9 1.06
COULERCI AI TIRE TREAD

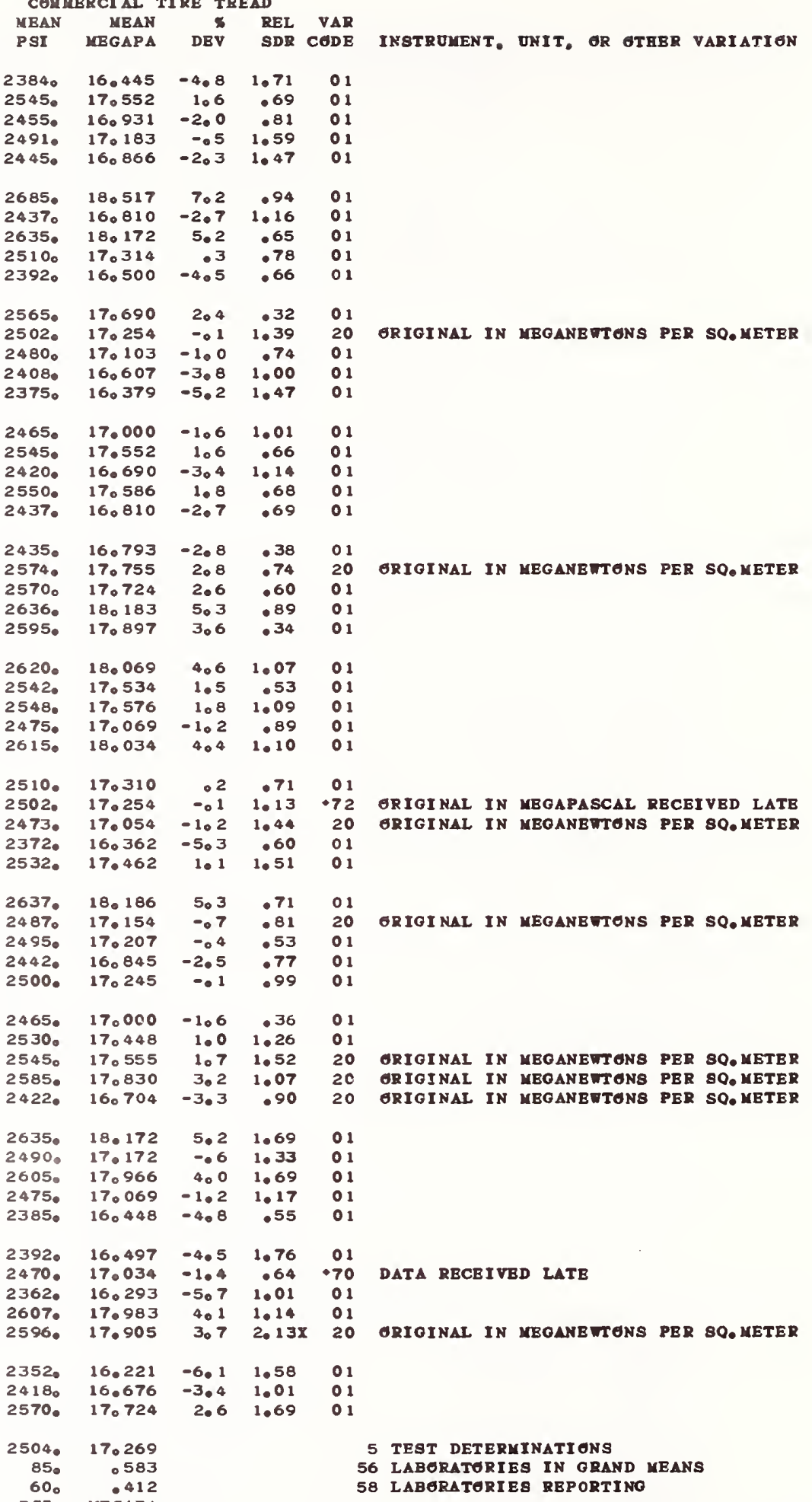

PSI

MEGAPA 
TENSILE STRENGTH

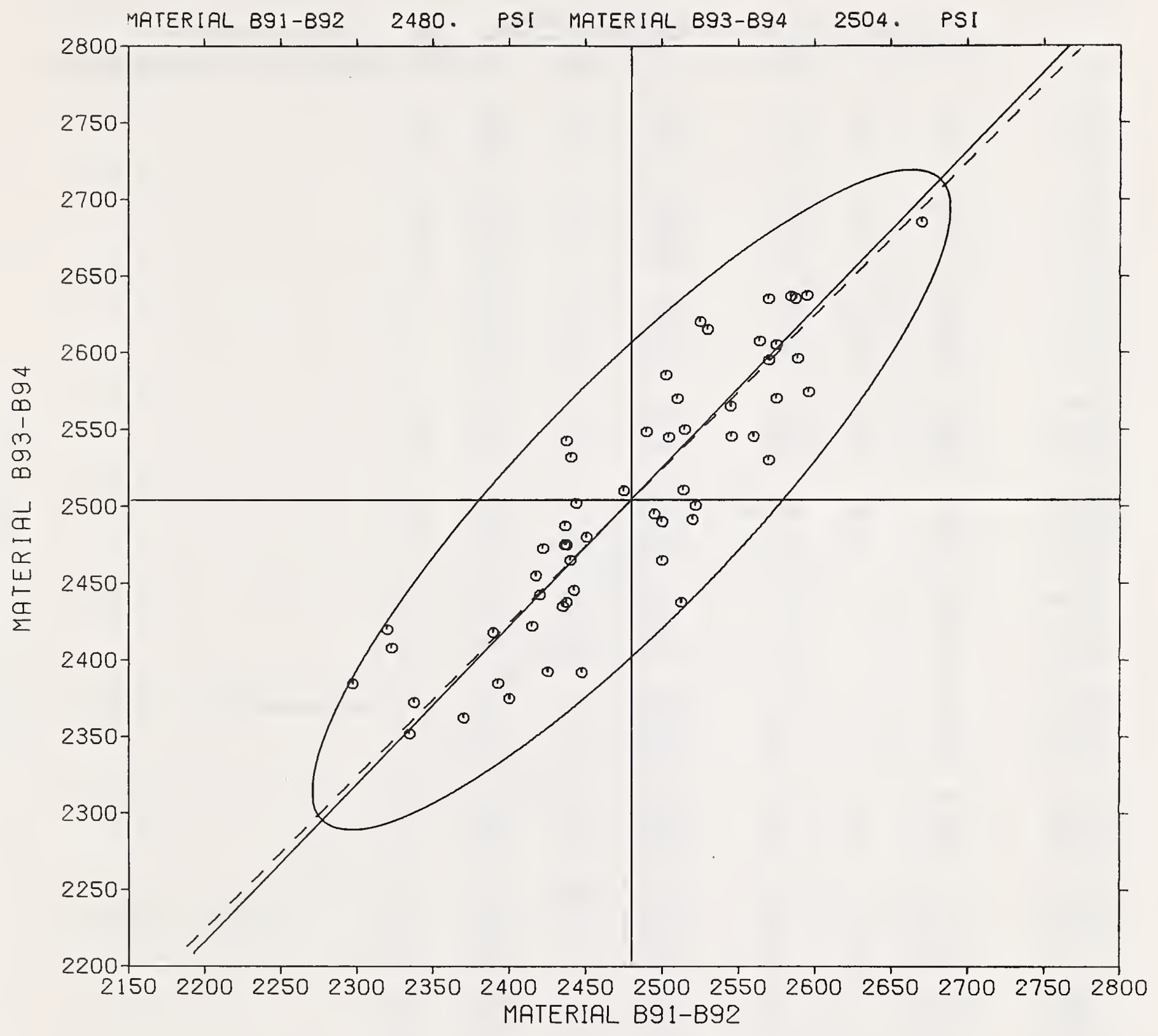


INTBRLABERATORY PROORAY ON BVALUATIGN GF RUBBER

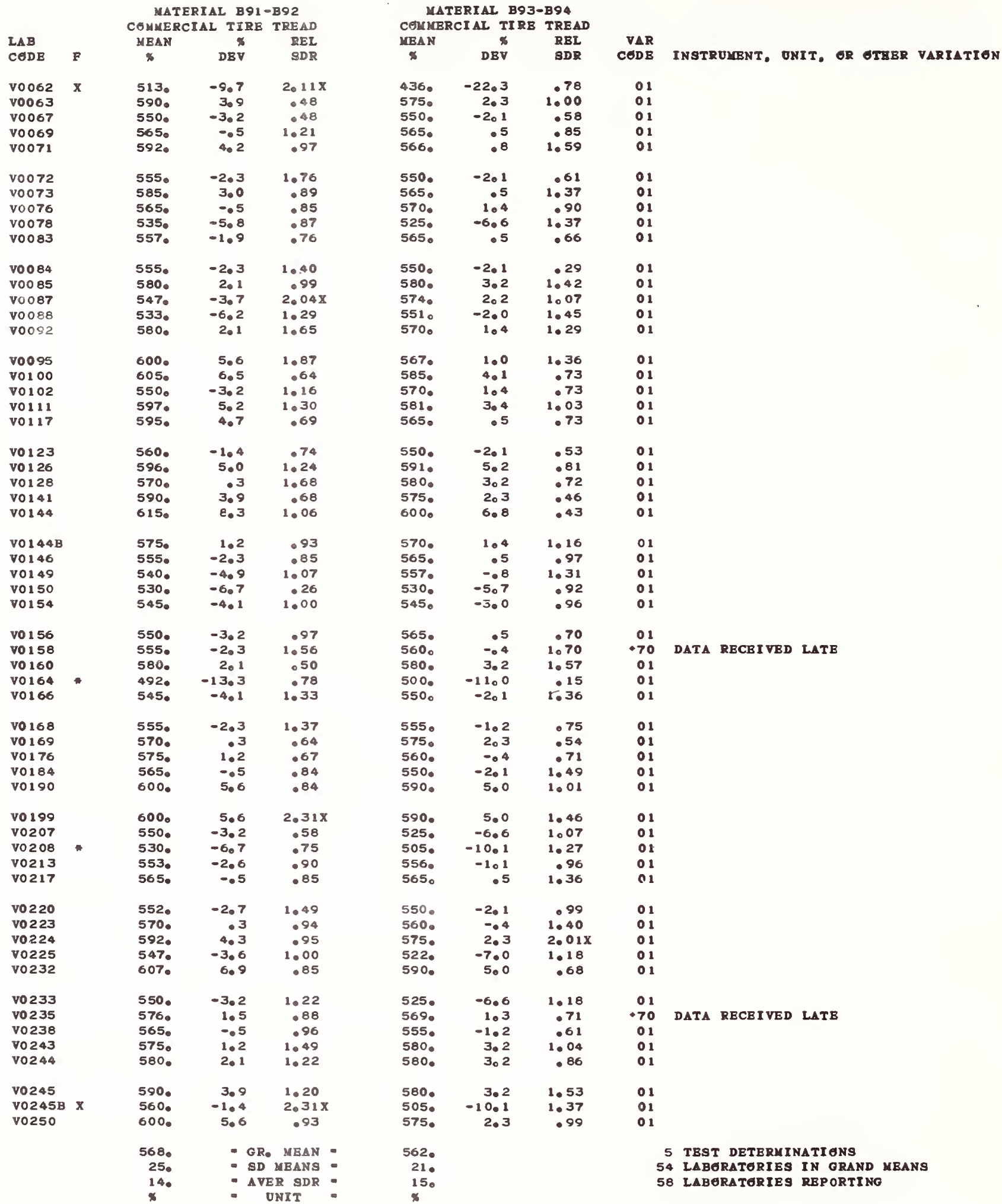




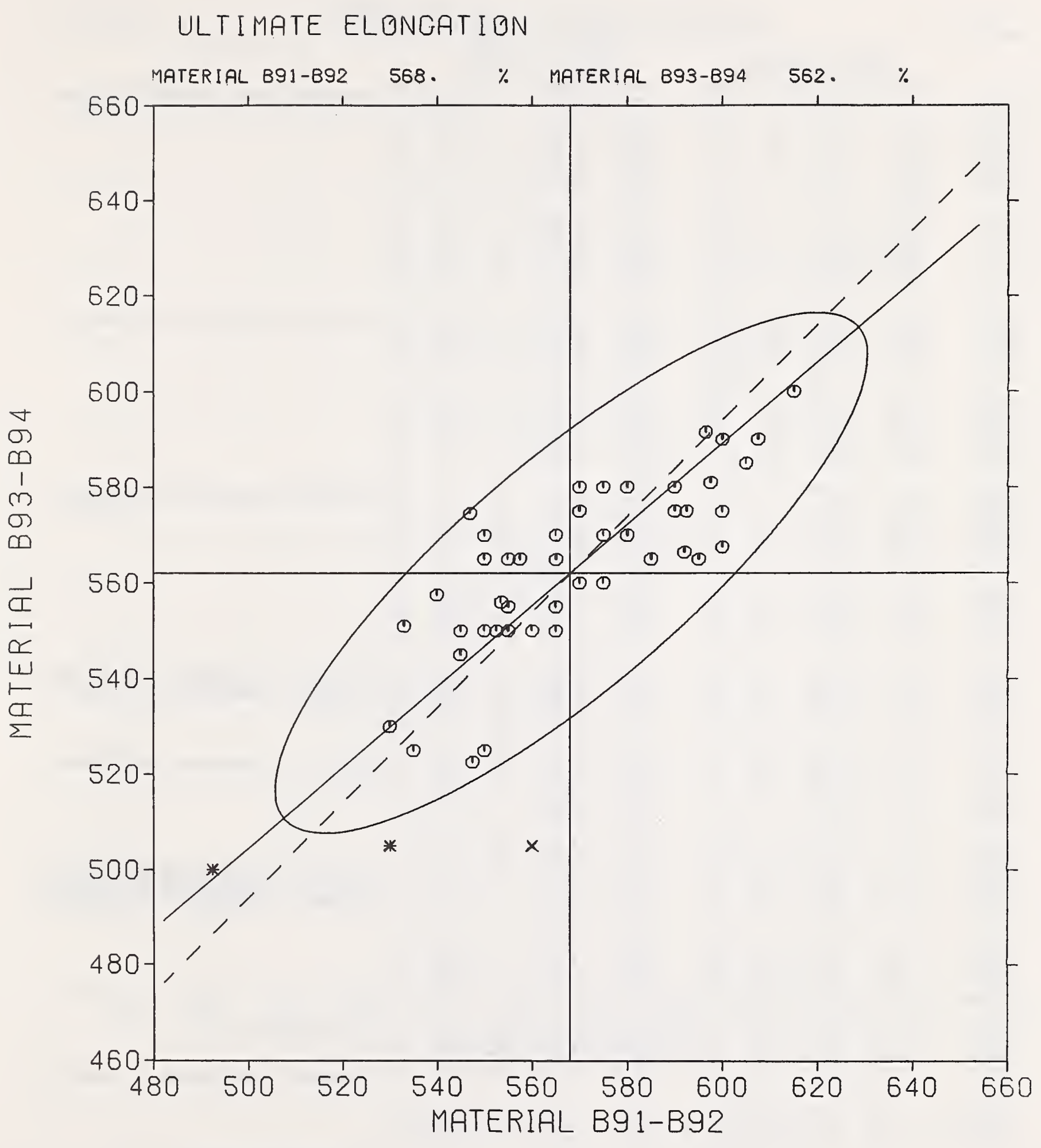


MATETIAL B91-B92

CPMNERCIAL TIRE TREAD

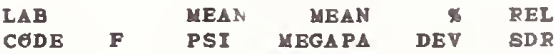

v0067

v0069

vo071

vo0 72

v0073

voo 0076

i. 078

v0083

vo 084

v0085

voony

vooes

vo09?

v0095

vo 100

v0102

vo 111

vo117

vo 123

Vo 126

vo 128

vo 141

VO144

V0 $144 \mathrm{~B}$

Vo1 146

vo 149

vo150

vo154

vo 156

vo 158

v0160

vo164

vo 166

vo 168

v0169

vo1 $70^{\circ}$

vo1 84

vo1 90

v0 199

vo 207

v020

vo2 13

vo214

v0 220

v0223

v0224

v0225

v0232

vo233

vo 235

v0238

vo 243

vo244

ve $245 A$

vO245B

vo 250

$8.700-1.5 \quad 1.75$

$8.383 \quad-5,1 \quad .72$

$8.724=1.3 \quad 1.36$

$8.534 \quad-3.4 \quad 1.52$

$9.276 \quad 5=0 \quad .91$

$9.3726 .1 \quad .64$

1295. $8.934 \quad 1.11 .91$

$1185 . \quad 8.172 \quad-7.5 \quad .74$

1227.

$8.466-4.2 \quad 1.01$

$\begin{array}{llll}1295 . & 8.241 & -6.7 & 1.03\end{array}$

1212. $\quad 8,362 \quad-5,4 \quad 1.26$

1260. $8.690-1.6 \quad 1.09$

1207.

1280.

$8.828 \quad-1 \quad .51$

1329. $9.166 \quad 3.7 .35$

$\begin{array}{rrr}9.166 & 3.7 & 035 \\ 8.345 & -5.6 & .51\end{array}$

8.862 .3 .41

1285

1242.

13440

1287.

1365 .

4.91 .79

.5 $\quad 079$

1290. 8.897 $\quad 7 \quad \div 88$

9.20

8.752

.09 .73

$1282.8 .245 \quad 1 \quad 1.18$

$8.738-1.1 \quad .75$
MATERIAL B93-B94

COUMBRCIAL TIRE TREAD

MEAN MEAN 5 REL VAR

PSI NEGAPA DEV SDR CEDB INSTRUMENT, UNIT, OR GTHER VARIATION

$12820 \quad 8.845 \quad-00 \quad 072 \quad 01$

1272. $8.776 \quad 08 \quad .94 \quad 01$

1257. $8,672 \quad-2,0 \quad 2,13 \mathrm{X} \quad 01$

1250. $8.624 \quad-2.5 \quad .43 \quad 01$

1245.

1175.

1345 .

1363.

1200.

$8.586-3.0 \quad .90 \quad 01$

$8.103 \quad-8.4 \quad 1.18 \quad 01$

$9.276 \quad 4.8 \quad .54 \quad 01$

$9.400 \quad 6.2 \quad 2.13 \times \quad 01$

8. $276-6.5 \quad .90 \quad 01$

1305. $9.000 \quad 1.7 \quad 1.30 \quad 01$

$\begin{array}{lllll}1247 . & 0.602 & -2.8 & 1.91 & 20 \\ 1268 & 0.745 & -1.2 & 1.90 & 01\end{array}$

1247. $\quad 8.600 \quad-2.8 \quad 2.73 \times \quad 01$

1165. $8.034 \quad-9.2 \quad 1.56 \quad 01$

1227

$8.466 \quad-4.3 \quad 1.56 \quad 01$

1230. $8.483 \quad-4.1 \quad 1.09 \quad 01$

1185. $8.172 \quad-7.6 \quad 1.35 \quad 01$

1255. $8.655-2.2 \quad .82 \quad 01$

1290.

1203.

1310.

1355.

1235.

1335.

1267.

1291.

1305 .

1385.

8. 897

$\begin{array}{rrr}8.897 & .6 & .75 \\ 8.297 & -6.2 & .65\end{array}$

$034-201-74$

$9.345 \quad 5.6 \quad .59$

$8.517 \quad-3.7 \quad .60$

$9.207 \quad 4.1 \quad .41$

$8.741-1.2 \quad 1.21$

$8.907 \quad 07 \quad 1.03$

$9.000 \quad 1.7 \quad .62$

$9.552 \quad 8.0 \quad 1.03$

1270.

$-1.0$

$2.50 x$

16.11 .27

$9.010 \quad 2.0 \quad 1.09$

1284

8.852

$8.802 \quad-.5 \quad .86 \quad 20$

.1

.5901

$1490 \% 10.279 \quad 16.2 \quad 1.13 \quad 01$

$9.366 \quad 5.9 \quad 1.23 \quad 01$

1336.

$9.214 \quad 4.1 \quad .61 \quad 01$

$8.452 \quad-4.5 \quad .38 \quad 20$

$9.241 \quad 4.5 \quad 1.02 \quad 01$

$8.862 \quad .21 .61 \quad 01$

$8.497 \quad-4.0 \quad 1.28 \quad 01$

$8.638 \quad-2.2 \quad 2.18 x$

1257.

$\begin{array}{llll}8.672 & -2.0 & 1.52 & 01\end{array}$

1415. $9.75910 .3 \quad 1.64$

1323. $9.127 \quad 3.2 \quad 1.53 \quad 20$

$1291.8 .902 .6 \quad .3920$

$\begin{array}{lllll}13100 & 9.034 & 2.1 & .58 & 01\end{array}$

$\begin{array}{lllll}1265 . & 8.724 & -1.4 & 1.17 & 01\end{array}$

$\begin{array}{rrrcr}1375 . & 9.483 & 7.2 & 2.06 \times & 01 \\ 1132 . & 7.810-11.7 & .64 & 01\end{array}$

1278. $8.817 \quad-.3 \quad 1.1101$

1250. $8.624 \quad-2.5 \quad .68 \quad 70$

$\begin{array}{lllll}1162 . & 8.017 & -9.4 & 1.35 & 01\end{array}$

1266. $8.731-1.3 \quad .84 \quad 01$

$13010 \quad 8.972 \quad 1.4 \quad 2.14 \times 20$

1109. $7.648-13.6 \quad 2.14 X \quad 01$

$\begin{array}{lllll}1317 . & 9.086 & 2.7 & 2.391 & 01\end{array}$

1275.

8.793

$\because 6$

1.1901

1283.8 .847

.523

.163

MEGAPA
GRIOINAL IN MBGAPASCAL RECEIVED LATE GRIGINAL IN MEGANETTENS PER SQ.METER

ORIGINAL IN MBGANETTGNS PBR SO, METER

ORIOINAL IN MEGANBWTENS PBR SO.METER

GRIOINAL IN UBOANEWTONS PER SQ.METER

ORIGINAL IN MEGANEWTONS PER SQ,METER

GRIGINAL IN MEGANEUTGNS PER SQ,METER

GRIGINAL IN MEGANBUTUNS PER SO, MBTER

DATA RECEIVED LATE

5 TEST DETERMINATIONS

55 IABORATERIES IN GRAND MEANS

58 IABERATERIES REPORTING
GRIGINAL IN MBGANETTONS PER SO, YETER 
STRESS AT 300\% ELONGATION

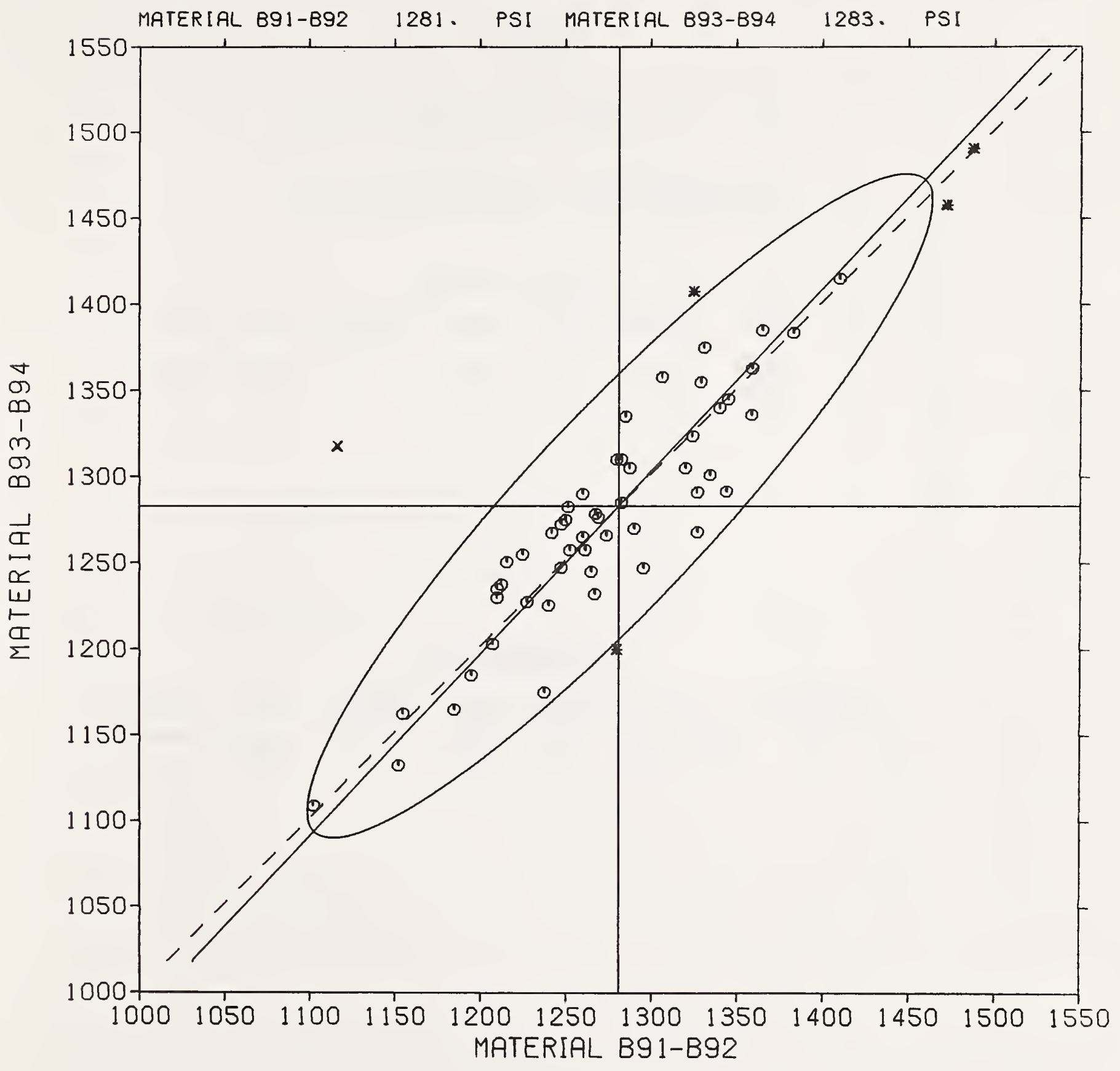





\section{HARDNESS}

NOTES

Materials B91 and $B 92$ were sheets of the same vulcanized rubber. Similarly, materials B93 and B94 were alike.

V100 results were obtained at NBS using ASTM D1415.

Four of the 28 participants reporting used ASTM D1415 (Wallace) for the hardness determination. A11 others used ASTM D2240 (Type A Durometer).

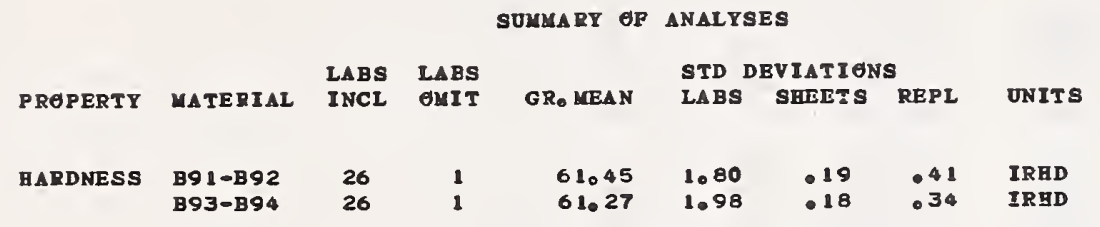

PRECISIGN OF METHEDS

\begin{tabular}{|c|c|c|c|c|c|c|c|c|c|}
\hline \multirow[b]{2}{*}{ P RE PERT Y } & \multirow[b]{2}{*}{ MATERIAI } & \multirow{2}{*}{$\begin{array}{r}\text { REPL } \\
\text { CRP }\end{array}$} & \multirow{2}{*}{$\begin{array}{l}\text { REPL } \\
\text { ASTM }\end{array}$} & \multicolumn{4}{|c|}{ ABSELUTB } & \multicolumn{2}{|c|}{ PERCENT } \\
\hline & & & & GR, MEAN & REPEAT & REPRED & DNITS & REPEAT & REPRED \\
\hline HARDNESS & $\begin{array}{l}B 91-B 92 \\
B 93-B 94\end{array}$ & $\begin{array}{l}5 \\
5\end{array}$ & $\begin{array}{l}5 \\
5\end{array}$ & $\begin{array}{l}61.45 \\
61.27\end{array}$ & $\begin{array}{r}1.14 \\
.93\end{array}$ & $\begin{array}{l}4.98 \\
5.50\end{array}$ & $\begin{array}{l}\text { I RHD } \\
\text { I RHD }\end{array}$ & $\begin{array}{l}1.9 \\
1.5\end{array}$ & $\begin{array}{l}8.1 \\
900\end{array}$ \\
\hline
\end{tabular}


INTERLABERATERY PREGRAM ON EVALUATION GF RUBBER

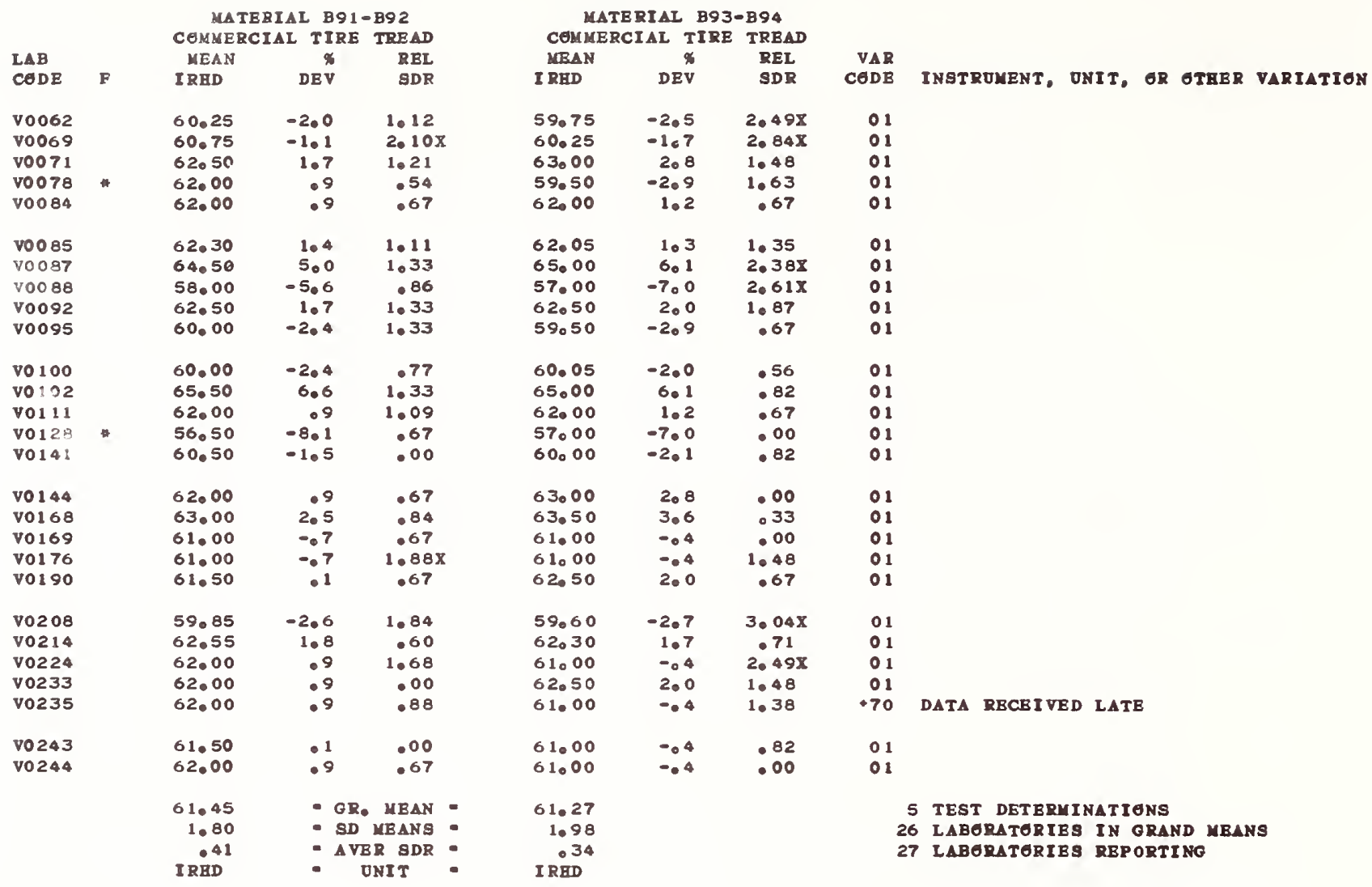


HARDNESS

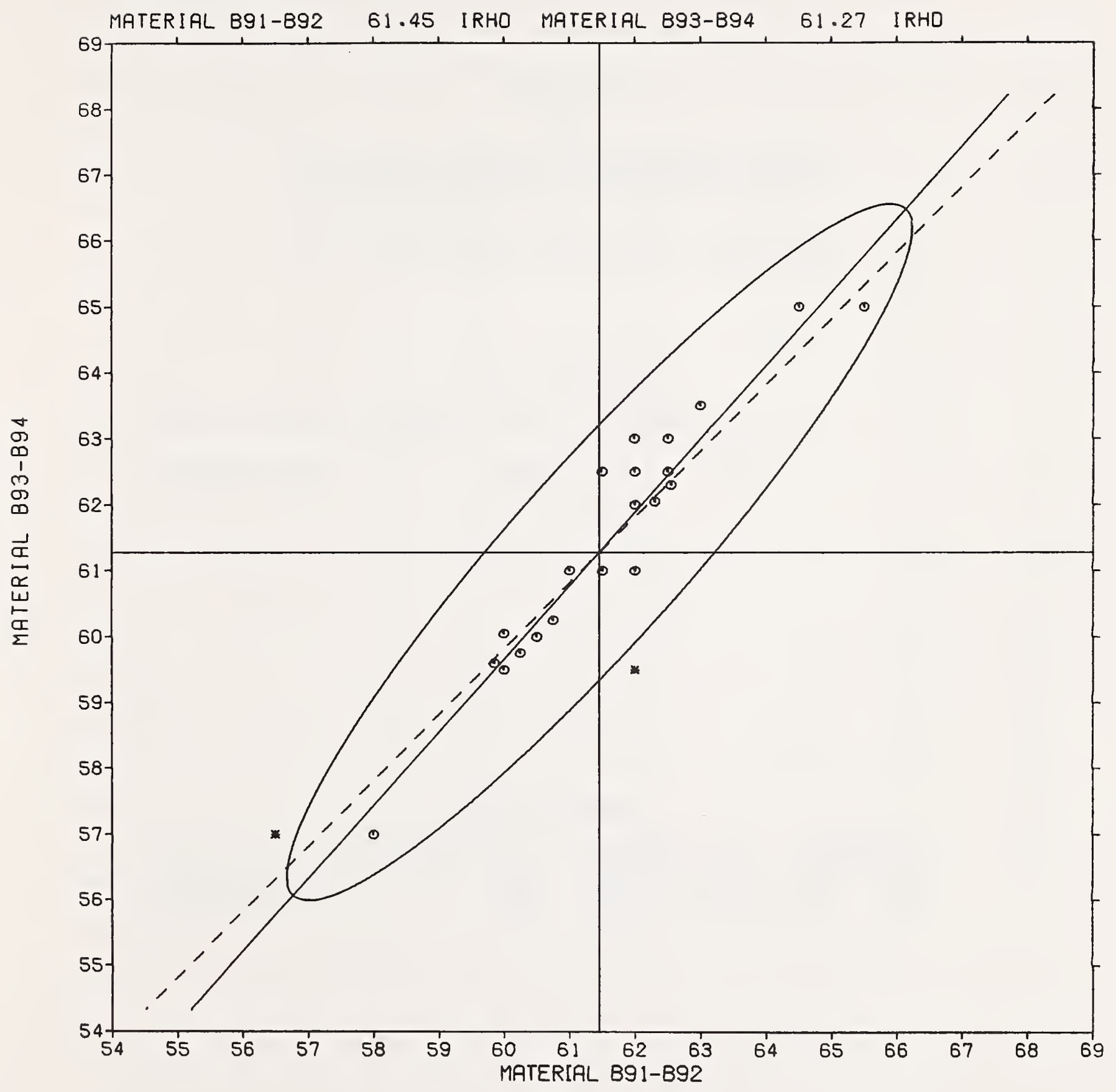



MOONEY VISCOSITY

NOTES

Materials $\mathrm{S} 91$ and $\mathrm{S} 92$ were the same rubber. Similarly, materials S93 and S94 were the same rubber. No sample preparation was required for materials $\mathrm{S} 91$ and $\mathrm{S} 92$ whereas, mill massing was required for materials $\mathrm{S} 93$ and $\mathrm{S} 94$.

V100 results were obtained at NBS on the manually closed viscometer used for determining the Mooney viscosities of the standard rubbers.

SUMKARY OF ANALYSES

\begin{tabular}{|c|c|c|c|c|c|c|c|c|}
\hline & & LABB & LABS & & STD & BVTATICN & & \\
\hline PREPERTY & MATEBIAL & INCL & OXIT & GR, MEAN & LABS & \&HEETS & REPL & NIT \\
\hline $\begin{array}{r}\text { MeबNBY } \\
\text { I ScesITY }\end{array}$ & $\begin{array}{l}891-892 \\
993-\$ 94\end{array}$ & $\begin{array}{l}40 \\
40\end{array}$ & $\begin{array}{l}3 \\
3\end{array}$ & $\begin{array}{l}67.53 \\
64.60\end{array}$ & $\begin{array}{l}2.00 \\
3.04\end{array}$ & $\begin{array}{l}.15 \\
.57\end{array}$ & $\begin{array}{l}.37 \\
.63\end{array}$ & \\
\hline
\end{tabular}

PRECISION OF METHEDS

\begin{tabular}{|c|c|c|c|c|c|c|c|c|c|}
\hline \multirow[b]{2}{*}{$\mathbf{T Y}$} & \multirow[b]{2}{*}{ MATERIAL } & \multirow{2}{*}{$\begin{array}{r}\text { REPL } \\
\text { CRP }\end{array}$} & \multirow{2}{*}{$\begin{array}{l}\text { REPL } \\
\text { ASTM }\end{array}$} & \multirow[b]{2}{*}{ GR, MEAN } & \multicolumn{2}{|c|}{ ABSOLUTE } & \multirow[b]{2}{*}{ UNITS } & \multicolumn{2}{|c|}{ PERCENT } \\
\hline & & & & & REPBAT & REPRED & & REPEAT & REPR GD \\
\hline $\begin{array}{r}\text { MUUNEY } \\
\text { vIscesITY }\end{array}$ & $\begin{array}{l}891-892 \\
993-894\end{array}$ & $\begin{array}{l}3 \\
3\end{array}$ & $\begin{array}{l}3 \\
3\end{array}$ & $\begin{array}{l}67.53 \\
64.60\end{array}$ & $\begin{array}{l}1.03 \\
1.75\end{array}$ & $\begin{array}{l}\text { 5. } 53 \\
8.42\end{array}$ & $\frac{12}{42}$ & $\begin{array}{l}1.5 \\
2.7\end{array}$ & $\begin{array}{r}8.2 \\
13.0\end{array}$ \\
\hline
\end{tabular}


INTERLABGRATERT PROGRAY GN EVALUATIGN GF RUBBER

\begin{tabular}{|c|c|c|c|c|c|c|c|c|c|c|c|}
\hline \multirow{3}{*}{$\begin{array}{l}\mathrm{LAB} \\
\mathrm{C} D \mathrm{DB}\end{array}$} & \multicolumn{5}{|c|}{ MATERIAL $\$ 91-\$ 92$} & MA & MATERIAL $993-\$ 94$ & $-\$ 94$ & \multirow[b]{2}{*}{ VAR } & \multirow[b]{3}{*}{ INSTROXENI, UNIT, OR OTBBR V } & \multirow[b]{3}{*}{ VARIATION } \\
\hline & & MEAN & x & REL & & MEAN & \% & RBL & & & \\
\hline & $\mathbf{F}$ & ML & DBV & SDR & & NII. & DBV & EDR & CEDE & & \\
\hline v0060 & & 67.00 & .08 & - 39 & & 66.00 & $2 \cdot 2$ & - 83 & 01 & & \\
\hline vo061 & & 66.80 & -1.1 & .34 & & 66.55 & 3.0 & .36 & 01 & & \\
\hline vo068 & & 72.00 & 6.6 & $4.93 x$ & & 69.50 & 7.6 & 2.00 & 01 & & \\
\hline vo071 & & 68.65 & 1.7 & .54 & & 68,35 & 5.8 & .82 & 01 & & \\
\hline vo072 & & 69.00 & 2.2 & 1.16 & & 64.50 & -02 & 1.37 & 01 & & \\
\hline vop 73 & & 65.00 & -3.7 & 1.55 & & 63.50 & $-1 \cdot 7$ & 1.67 & 01 & & \\
\hline vo077 & $x$ & 66.05 & -2.2 & 1.05 & & 47.80 & -26.0 & .35 & 01 & & \\
\hline voo 78 & $\mathbf{x}$ & 60.00 & -11.1 & 1.55 & & 55.50 & -14.1 & 1.39 & 01 & & \\
\hline vo079 & & 67.15 & -.6 & 1.02 & & 64.30 & -.5 & 1.39 & 01 & & \\
\hline voo 80 & & 67.00 & -.8 & 1.78 & & 66.55 & 3.0 & 1.10 & 01 & & \\
\hline vo0 83 & & 69.05 & $2 \cdot 3$ & 1.32 & & 68,25 & 5.7 & 2.01 & 01 & & \\
\hline vo0 85 & & 72.50 & 7.4 & 1.45 & & 70.25 & 8.8 & .46 & 01 & & \\
\hline vo09o & & 69.75 & $3 \cdot 3$ & .77 & & 63.75 & -1.3 & - 23 & 01 & & \\
\hline vo092 & & 67.00 & -.8 & 1.55 & & 67.00 & 3.7 & .00 & 01 & & \\
\hline voo95 & & 68.75 & 1.8 & 1.06 & & 64.75 & .2 & 1.60 & 01 & & \\
\hline vo 100 & & 68.75 & 1.8 & .50 & & $65 \cdot 25$ & 1.0 & .46 & 01 & & \\
\hline vo 111 & * & 63.50 & -6.0 & .77 & & 56.00 & -1303 & 1.21 & 01 & & \\
\hline vo 117 & & 66.25 & -1.9 & 1.55 & & 63.00 & -2.5 & 1.21 & 01 & & \\
\hline VO 128 & & 70.50 & 4.4 & .77 & & 68,50 & 6.0 & 1.58 & 01 & & \\
\hline V01 44 & & 68.75 & 1.8 & .92 & & $64 \cdot 25$ & -.5 & . 32 & 01 & & \\
\hline vo 146 & & 65.00 & -3.7 & .39 & & 62.50 & $-3 \cdot 2$ & .00 & 01 & & \\
\hline VO 149 & & 68.65 & 1.7 & .95 & & $65 \cdot 45$ & $1 \cdot 3$ & .60 & 01 & & \\
\hline vo 150 & & 67.50 & -00 & .77 & & 64.00 & -09 & 2.04 & 01 & & \\
\hline vo 156 & & 66.00 & -2.3 & .39 & & 65.75 & 1.8 & .40 & 01 & & \\
\hline V0166 & & 66.00 & -2.3 & .39 & & 64.25 & -.5 & .92 & 01 & & \\
\hline vo 177 & & 66.55 & -1.4 & 1.25 & & 67.35 & 4.3 & 1.26 & 01 & & \\
\hline VO 182 & & 67.50 & .0 & 77 & & $63 \cdot 25$ & -201 & .46 & 01 & & \\
\hline vo 1 Sc & & 70.00 & 3.7 & .84 & & 63.90 & -1.1 & 88 & 01 & & \\
\hline vo 207 & & 68.90 & 2.0 & .66 & & 62.70 & -2.9 & .40 & 01 & & \\
\hline vo 208 & & 67.00 & -.8 & 1.55 & & 60.50 & -6.3 & 1.58 & 01 & & \\
\hline 70213 & & 66.50 & -1.5 & 1.16 & & 63.75 & $-1 \cdot 3$ & .63 & 01 & & \\
\hline vo 214 & & 67.00 & -88 & 1.41 & & 65.00 & .6 & .85 & 01 & & \\
\hline vo 217 & & 64.00 & -5.2 & 39 & & 61.75 & -4.4 & .69 & 01 & & \\
\hline vO 218 & & 68.50 & 1.4 & .77 & & 66.00 & 2.2 & - 92 & 01 & & \\
\hline vo 220 & & 65.85 & -2.5 & .96 & & 64.65 & .1 & $2 \cdot 89 x$ & 01 & & \\
\hline vo221 & & 68.60 & 1.6 & 1.07 & & 67.35 & $4 \cdot 3$ & .45 & 01 & & \\
\hline vo223 & & 66.00 & $-2 \cdot 3$ & .77 & & 64.50 & -.2 & - 92 & 01 & & \\
\hline vo 230 & & 66.05 & $-2 \cdot 2$ & .69 & & 58.50 & -9.4 & .32 & 01 & & \\
\hline vo 236 & & 69.00 & 2.2 & 2.12 & & 64.50 &. .2 & 2.00 & 01 & & \\
\hline $\mathbf{v 0 2 3 8}$ & - & 64.00 & $-5 \cdot 2$ & 2.12 & & 56.50 & -12.5 & - 92 & 01 & & \\
\hline vo 244 & & 66.00 & -2.3 & 1.55 & & 65.00 & .6 & 1.83 & $\bullet 70$ & DATA RECEIVED LATE & \\
\hline vo 250 & & 68.50 & 1.4 & .77 & & 67.50 & 4.5 & .92 & 01 & & \\
\hline vo 251 & & 66.60 & -1.4 & 1.35 & & 64.00 & .9 & $1 \cdot 24$ & 01 & & \\
\hline & & 67.53 & - c & - MEAN & - & 64.60 & & & $\mathbf{3}$ & TEST DETERMINATIENS & \\
\hline & & 2.00 & - $s$ & MEANS & - & 3.04 & & & 40 & LABGRATORIES IN GRAND MEANS & \\
\hline & & $i^{37}$ &. & ER SDR & - & .63 & & & 43 & LABGRATORIBS RBPORTING & \\
\hline
\end{tabular}


MOONEY VISCOSITY

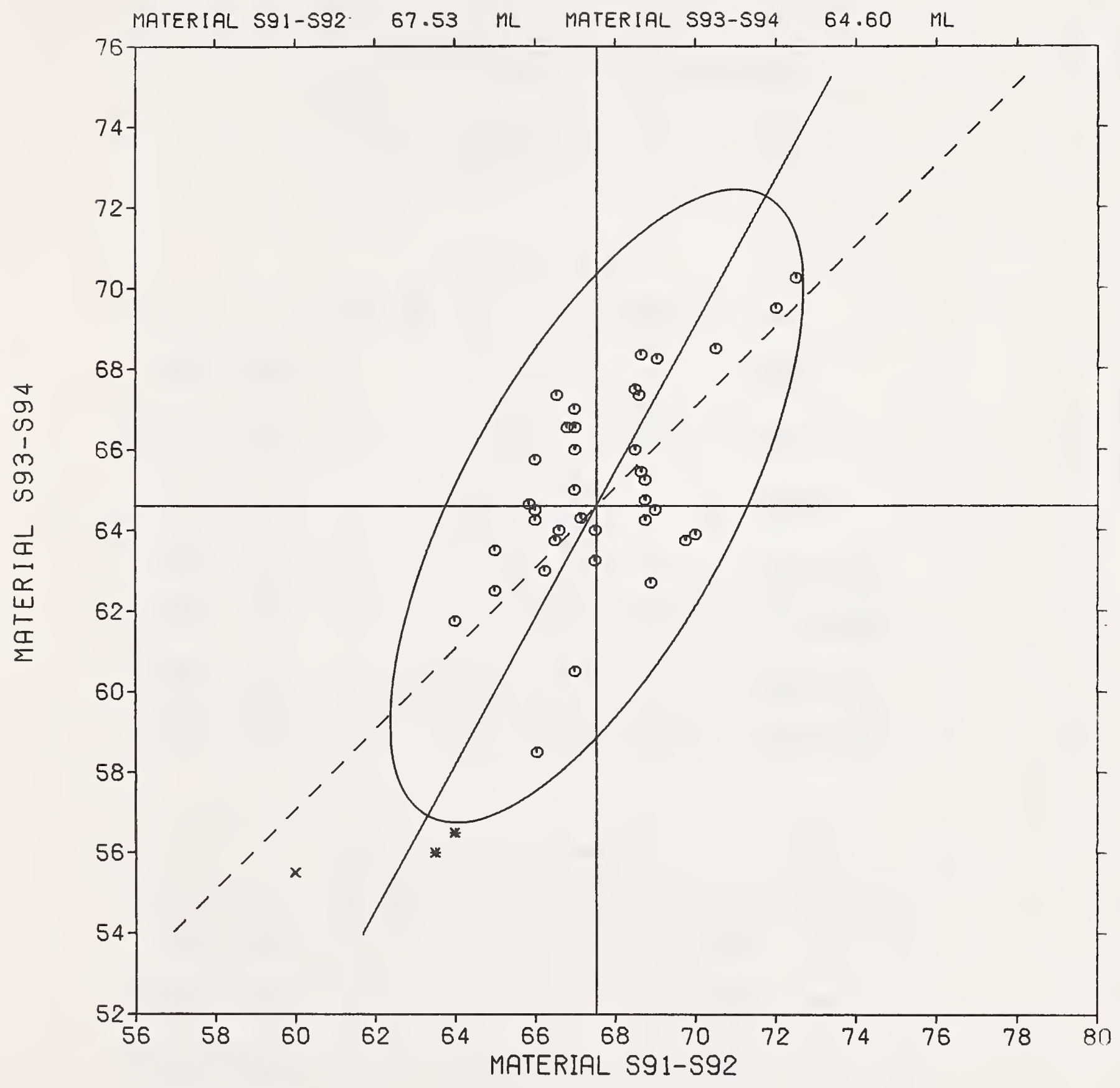



VULCANIZATION CHARACTERISTICS USING OSCILLATING DISK CURE IETER

\section{NOTES}

Materials $X 91$ and $X 92$ were the same rubber formulation. Similarly, materials $\mathrm{X93}$ and $\mathrm{X} 94$ were alike.

V100 results were obtained at NBS using a Model TM-100 Monsanto Rheometer with a disk oscillating at $+1^{\circ}$ amplitude and 1.7 hertz frequency.

All participants used Monsanto Rheometers operated at one degree amplitude and 1.7 hertz frequency.

Labs v0064 and v0074 reported calculation problems in Cure Time $(50 \%$ and $90 \% \mathrm{MH})$.

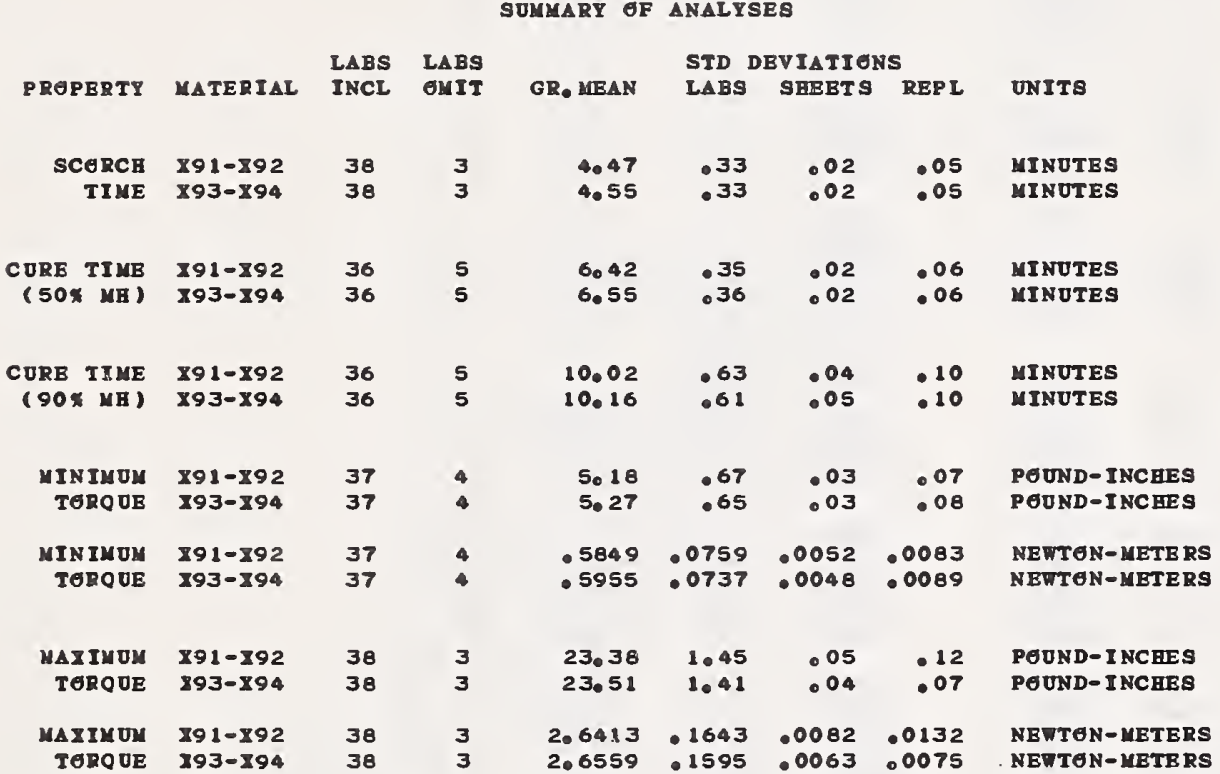

\begin{tabular}{|c|c|c|c|c|c|c|c|c|c|}
\hline \multirow[b]{3}{*}{ P REPERTY } & \multicolumn{9}{|c|}{ PRBCISION EF METHEDS } \\
\hline & \multirow[b]{2}{*}{ MATERIAL } & \multirow{2}{*}{$\begin{array}{l}\text { REPL } \\
\text { CRP }\end{array}$} & \multirow{2}{*}{$\begin{array}{l}\text { REPL } \\
\text { ASTM }\end{array}$} & \multirow[b]{2}{*}{ GR・MBAN } & \multicolumn{2}{|c|}{ ABSELUTE } & \multirow[b]{2}{*}{ UNITS } & \multicolumn{2}{|c|}{ PBRCENT } \\
\hline & & & & & REPEAT & RB PROD & & REPEAT & RBPRED \\
\hline SCERCH & $x 91-x 92$ & 3 & 3 & 4.47 & .15 & .90 & UINUTE & $3 \cdot 4$ & 20,2 \\
\hline IIMB & $x 93-x 94$ & 3 & 3 & 4.55 & .14 & .91 & MINUTE & 3. 1 & 19.9 \\
\hline $\begin{array}{c}\text { CURB TIMB } \\
\text { (50\% ME) }\end{array}$ & $\begin{array}{l}x 91-x 92 \\
x 93-x 94\end{array}$ & $\begin{array}{l}3 \\
3\end{array}$ & $\begin{array}{l}3 \\
3\end{array}$ & $\begin{array}{l}6.42 \\
6.55\end{array}$ & $\begin{array}{l}17 \\
.16\end{array}$ & $\begin{array}{r}.96 \\
1.00\end{array}$ & $\begin{array}{l}\text { MINUTE } \\
\text { MINUTE }\end{array}$ & $\begin{array}{l}2.6 \\
2.4\end{array}$ & $\begin{array}{l}14 \cdot 9 \\
15 \cdot 3\end{array}$ \\
\hline $\begin{array}{r}\text { CURE TIMB } \\
(90 \% \text { MH) }\end{array}$ & $\begin{array}{l}x 91-x 92 \\
x 93-x 94\end{array}$ & $\begin{array}{l}3 \\
3\end{array}$ & $\begin{array}{l}3 \\
3\end{array}$ & $\begin{array}{l}10.02 \\
10.16\end{array}$ & $\begin{array}{l}.28 \\
.27\end{array}$ & $\begin{array}{l}1.73 \\
1.69\end{array}$ & $\begin{array}{l}\text { MINUTE } \\
\text { MINUTE }\end{array}$ & $\begin{array}{l}2 \cdot 8 \\
2.6\end{array}$ & $\begin{array}{l}17.3 \\
16.7\end{array}$ \\
\hline $\begin{array}{l}\text { MINIM DN } \\
\text { TEROUE }\end{array}$ & $\begin{array}{l}x 91-x 92 \\
x 93-x 94\end{array}$ & $\begin{array}{l}3 \\
3\end{array}$ & $\begin{array}{l}3 \\
3\end{array}$ & $\begin{array}{l}5.18 \\
5.27\end{array}$ & $\begin{array}{l}20 \\
.22\end{array}$ & $\begin{array}{l}1.86 \\
1.81\end{array}$ & $\begin{array}{l}L B=I N_{0} \\
L B-I N_{0}\end{array}$ & $\begin{array}{l}3.9 \\
401\end{array}$ & $\begin{array}{l}35 \cdot 9 \\
34 \cdot 3\end{array}$ \\
\hline $\begin{array}{l}\text { WINIM UN } \\
\text { TEROOB }\end{array}$ & $\begin{array}{l}x 91-x 92 \\
x 93-x 94\end{array}$ & $\begin{array}{l}3 \\
3\end{array}$ & $\begin{array}{l}3 \\
3\end{array}$ & $\begin{array}{r}5849 \\
.5955\end{array}$ & $\begin{array}{l}.0229 \\
.0247\end{array}$ & $\begin{array}{r}.2102 \\
.2042\end{array}$ & $\begin{array}{l}N=M \\
N-M\end{array}$ & $\begin{array}{l}3.9 \\
4.1\end{array}$ & $\begin{array}{l}35.9 \\
34,3\end{array}$ \\
\hline $\begin{array}{l}\text { MAXINOX } \\
\text { TOROUE }\end{array}$ & $\begin{array}{l}x 91-x 92 \\
x 93-x 94\end{array}$ & $\begin{array}{l}3 \\
3\end{array}$ & $\begin{array}{l}3 \\
3\end{array}$ & $\begin{array}{l}23.38 \\
23.51\end{array}$ & $\begin{array}{r}-32 \\
.18\end{array}$ & $\begin{array}{l}4.03 \\
3.91\end{array}$ & $\begin{array}{l}L B-I N_{0} \\
L B-I N_{0}\end{array}$ & 1.4 & $\begin{array}{l}17.2 \\
16.6\end{array}$ \\
\hline $\begin{array}{r}\text { MAXIMUN } \\
\text { TOROUB }\end{array}$ & $\begin{array}{l}x 91-x 92 \\
x 93-x 94\end{array}$ & $\begin{array}{l}3 \\
3\end{array}$ & $\begin{array}{l}3 \\
\mathbf{3}\end{array}$ & $\begin{array}{l}2.6413 \\
2.6559\end{array}$ & $\begin{array}{l}.0366 \\
.0208\end{array}$ & $\begin{array}{r}.4552 \\
.4419\end{array}$ & $\begin{array}{l}N-M \\
N-M\end{array}$ & $\begin{array}{r}1.4 \\
.8\end{array}$ & $\begin{array}{l}17.2 \\
16 \cdot 6\end{array}$ \\
\hline
\end{tabular}


INTERLABERATERY PRGGRAM ON EVALUATION OF RUBBER

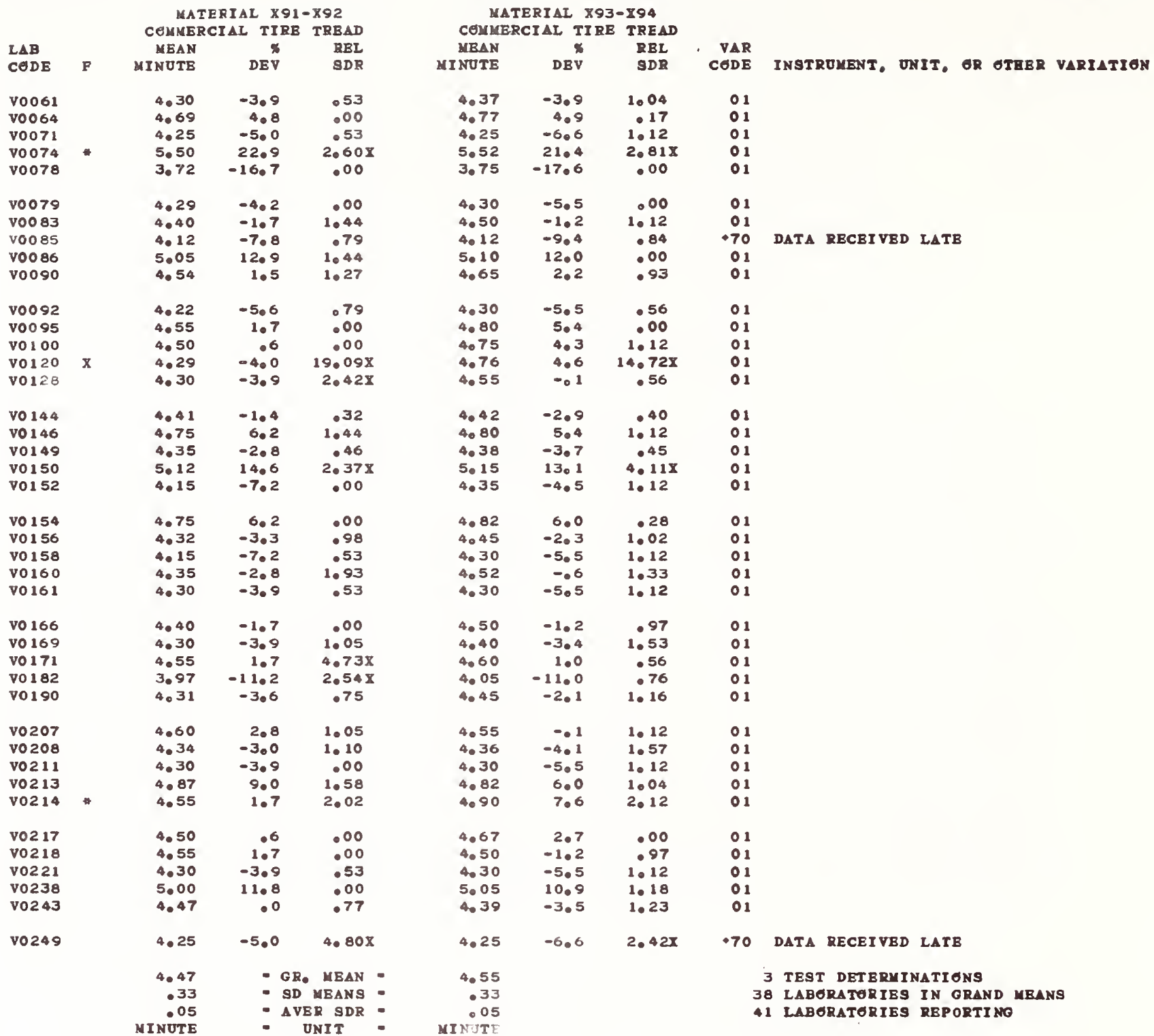


SCORCH TIME

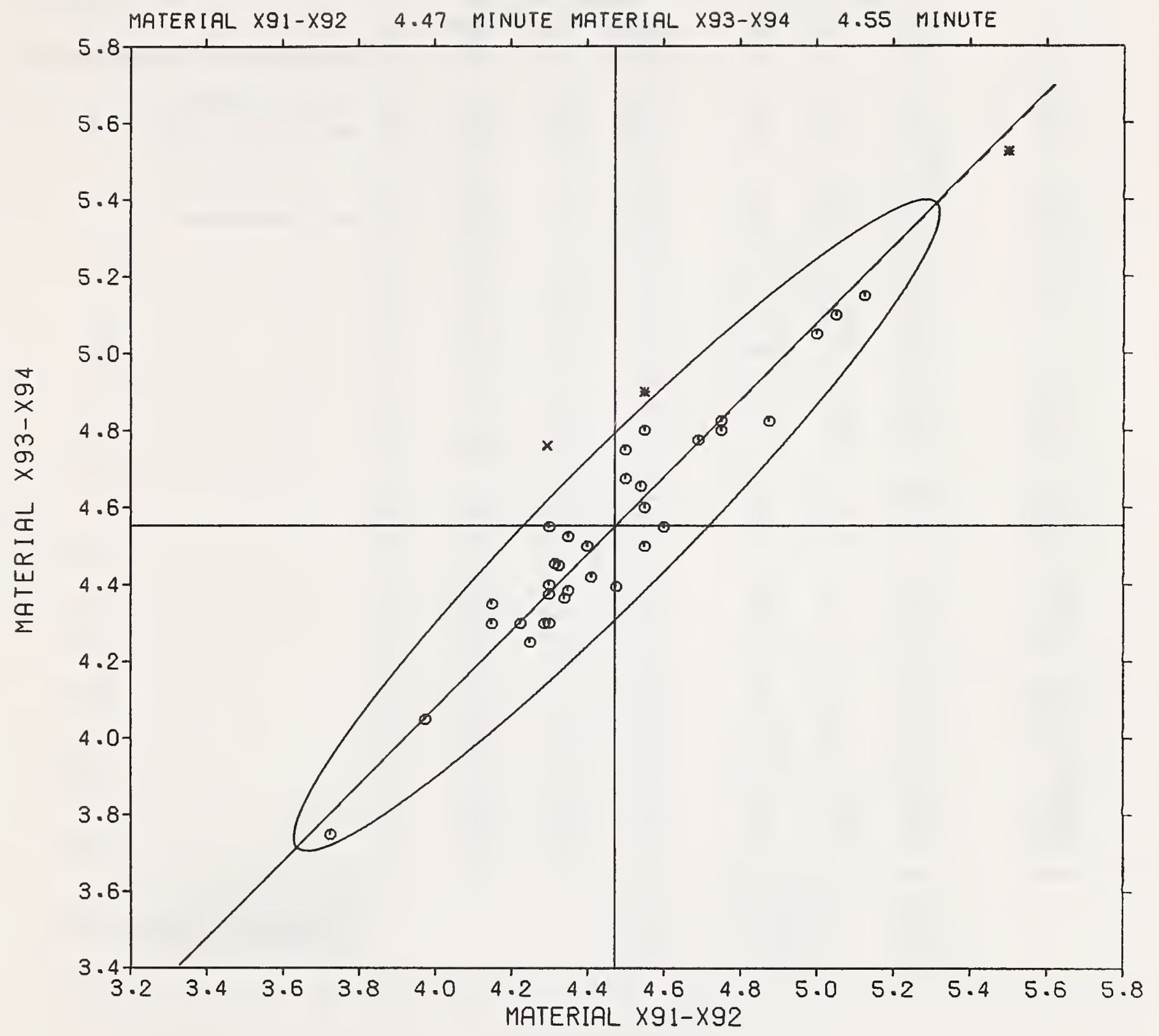


INTERLABERATERY PREGRAY ON BVALUATION OF RUBBER

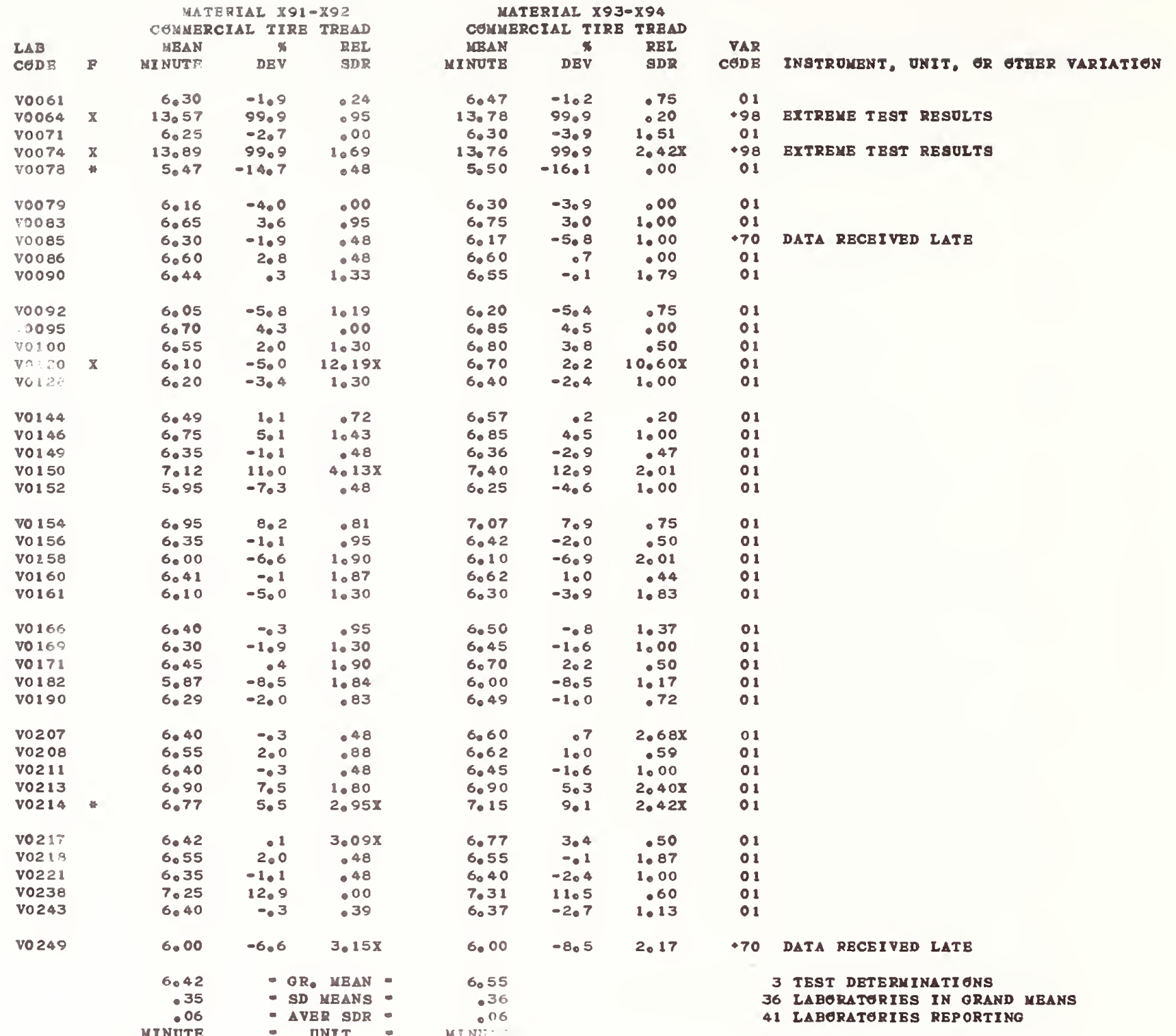




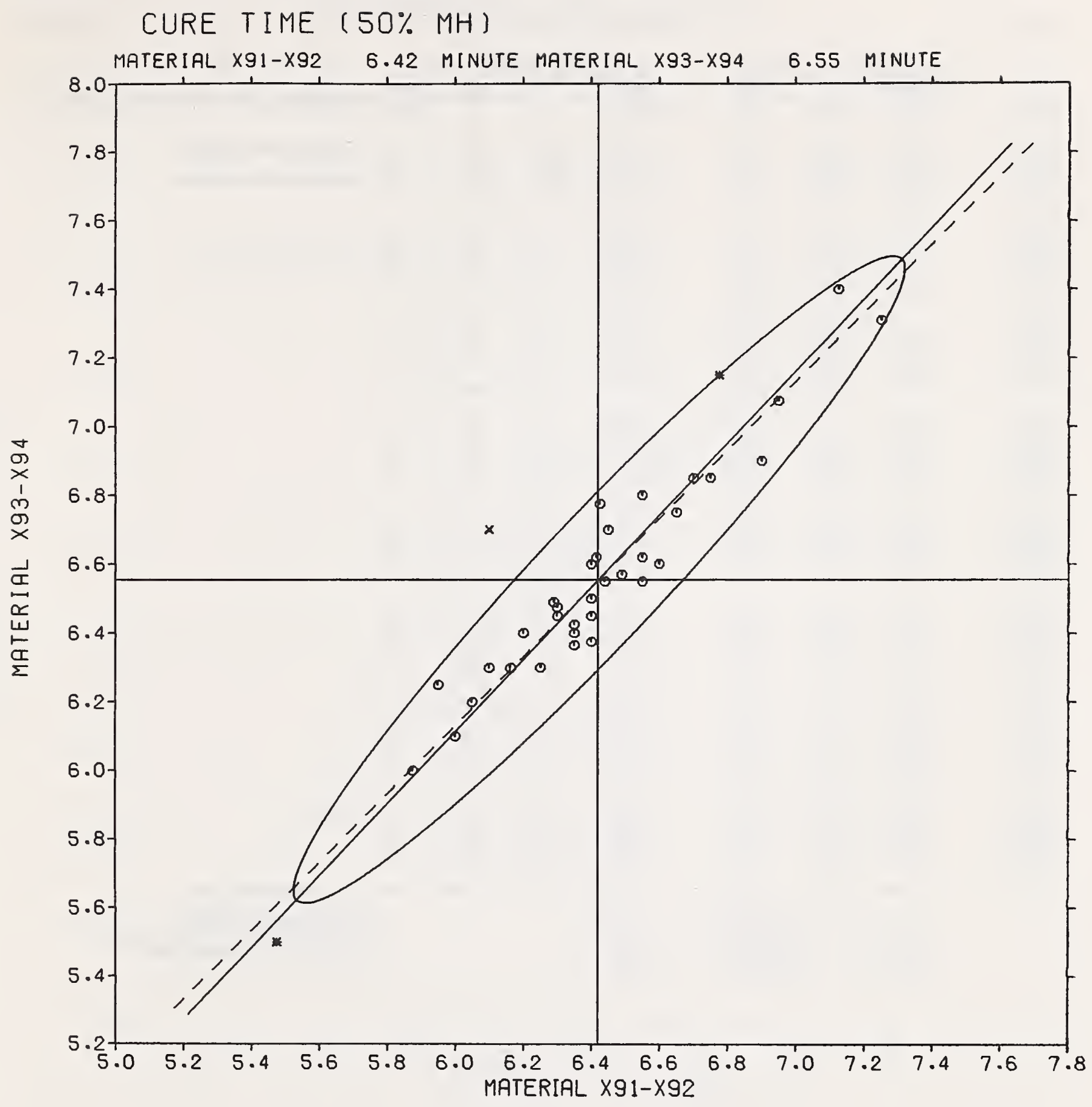




\begin{tabular}{|c|c|c|c|c|c|c|c|c|c|c|}
\hline & & $\begin{array}{r}\text { MAT } \\
\text { CEMMER }\end{array}$ & $\begin{array}{l}\text { IAL X91- } \\
\text { IAI IIRB }\end{array}$ & $\begin{array}{l}\text { X92 } \\
\text { TREAD }\end{array}$ & $\begin{array}{r}\text { MA } \\
\text { COMME }\end{array}$ & $\begin{array}{l}\text { BRIAL } X \\
\text { CIAL TI }\end{array}$ & $\begin{array}{l}3=X 94 \\
B \quad T R E A D\end{array}$ & & & \\
\hline$L A B$ & & MEAN & $\%$ & REL & MBAN & ๘ & REI & VAR & & \\
\hline $\operatorname{CODE}$ & $\mathbf{F}$ & NINUTE & DEV & SDR & MINUTE & DEV & SDR & CEDE & INSTRUMENT, UNIT, OR OTHER $V$ & VARIATIGN \\
\hline vo061 & & 9.82 & -200 & -28 & $10 \cdot 20$ & $\bullet$ & .82 & 01 & & \\
\hline$\nabla 0064$ & $\mathbf{X}$ & 20.49 & 99.9 & .80 & 20.77 & 99.9 & .20 & +98 & BXTREME TEET REBULTS & \\
\hline V0071 & & 9.80 & $-2,2$ & 57. & 9.85 & -3.0 & .90 & 01 & & \\
\hline v0074 & $\mathbf{x}$ & $21 \cdot 16$ & 99.9 & 1.35 & $21 \cdot 11$ & 99.9 & 1.72 & -98 & BXTREME TEST RBSULTB & \\
\hline vo 078 & & 8.57 & $-14 \cdot 4$ & 1. 23 & 8.60 & $-15 \cdot 3$ & $1 \cdot 28$ & 01 & & \\
\hline v0079 & & 9.36 & -6.6 & .00 & 9.79 & -3.6 & .00 & 01 & & \\
\hline vo 083 & & 10.70 & 6.8 & 1.14 & 10.75 & 5.8 & 1.12 & 01 & & \\
\hline 70085 & & 9.42 & -6.0 & 1.14 & 9.55 & -6.0 & .75 & +70 & DATA RECBIVED LATB & \\
\hline voo 86 & & 10.40 & $3 \cdot 8$ & 1.06 & 10.45 & 2.9 & .30 & 01 & & \\
\hline$\nabla 0090$ & & 9.98 & 0.4 & 1.29 & 10.04 & -1.2 & 1.53 & 01 & & \\
\hline vo092 & & 9.37 & -6.5 & 1.09 & 9.50 & -6.5 & . 30 & 01 & & \\
\hline vo095 & & 10.45 & $4 \cdot 3$ & .00 & 10.50 & 3.4 & .00 & 01 & & \\
\hline V0100 & & 10.70 & $6 \cdot 8$ & .78 & 10.70 & $5 \cdot 3$ & .60 & 01 & & \\
\hline vo 120 & & 10.37 & $3 \cdot 5$ & $12.05 x$ & 10.50 & 3.4 & $11.16 x$ & 01 & & \\
\hline vo i 28 & & 9.45 & -5.7 & 1.61 & 9.70 & $-4 \cdot 5$ & .60 & 01 & & \\
\hline vo 144 & & 10.00 & -.2 & .53 & 10.22 & .6 & .39 & 01 & & \\
\hline Vo 146 & & $10 \cdot 55$ & $5 \cdot 3$ & .49 & 10.50 & 3.4 & - 82 & 01 & & \\
\hline vo1 49 & & 9.77 & -2.5 & .52 & 9.87 & -2.8 & - 80 & 01 & & \\
\hline vo1 50 & & $11 \cdot 37$ & 13.5 & $2,66 x$ & 11.30 & 11.3 & 1.91 & 01 & & \\
\hline vo 152 & & 9.30 & -7.2 & .57 & 9.50 & -6.5 & .00 & 01 & & \\
\hline vo1 154 & & 11.17 & 11.5 & 1.00 & $11 \cdot 27$ & 11.0 & 1.01 & 01 & & \\
\hline V01 56 & & 9.85 & -1.7 & .75 & 10,00 & -1.5 & - 30 & 01 & & \\
\hline v0158 & & 9.40 & -6.2 & 1.42 & 9.40 & -7.5 & 1.50 & 01 & & \\
\hline vo1 60 & & 10.02 & .0 & 2.02 & 10.27 & 1.2 & .36 & 01 & & \\
\hline V0161 & & 9.50 & -5.2 & 1.48 & 9.90 & -2.5 & 1.20 & 01 & & \\
\hline vo 166 & & 9.60 & $-4 \cdot 2$ & .28 & 9080 & -3.5 & .00 & 01 & & \\
\hline vo 169 & & 9.75 & -2.7 & 1.06 & 10.00 & -1.5 & .30 & 01 & & \\
\hline vo 171 & $x$ & $9 \cdot 25$ & -7.7 & $3.69 x$ & 10.25 & - 9 & .60 & 01 & & \\
\hline vo 182 & & 9.22 & -8.0 & .89 & 9.25 & -8.9 & .96 & 01 & & \\
\hline vo 190 & & $10 \cdot 23$ & 2.1 & .83 & 10.46 & 3.0 & .57 & 01 & & \\
\hline vo 207 & & 10.35 & 3.3 & 1.24 & 10.85 & 6.8 & 1.70 & 01 & & \\
\hline 70208 & & 10.01 & -01 & 1.70 & 9.79 & -3.6 & 2.22 & 01 & & \\
\hline Vo 211 & & 10.00 & -.2 & 1.32 & 10.10 & .06 & 1.20 & 01 & & \\
\hline vo 213 & & 10.90 & 8,8 & 1.44 & 10.75 & 5.8 & 1.73 & 01 & & \\
\hline vo 214 & & 10.70 & 6.8 & $2.98 x$ & 11.20 & 10.3 & $2.75 x$ & 01 & & \\
\hline v0217 & & 9.50 & -5.2 & 1.42 & 10.12 & -.3 & .00 & 01 & & \\
\hline vo 218 & & 9.90 & -1.2 & .28 & 10.00 & -1.5 & 1.32 & 01 & & \\
\hline vo 221 & & 9.85 & $-1 \cdot 7$ & .57 & 9.70 & -4.5 & - 30 & 01 & & \\
\hline Y0238 & & $11 \cdot 25$ & 12,2 & 1.42 & 11.25 & 10.8 & 1. 50 & 01 & & \\
\hline vo 243 & & 9.61 & -4.1 & .70 & 9.55 & -6.0 & 1.43 & 01 & & \\
\hline vo249 & 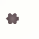 & 9.00 & $-10 \cdot 2$ & $4 \cdot 99 x$ & 8.75 & -13.9 & $3 \cdot 76 x$ & 470 & DATA RECEIVED LATE & \\
\hline & & 10.02 & - GRo & MBAN & 10.16 & & & & 3 TBET DETERMINATIGNA & \\
\hline & & .63 & - $8 D$ & МВANS & .61 & & & & 6 LABGRATERIEB IN ORAND MBANB & \\
\hline & & $\cdot 10$ & - AVEI & SDR & $\cdot 10$ & & & & 1 IABGRATORIES REPORTINO & \\
\hline
\end{tabular}


CURE TIME ( $90 \% \mathrm{MH})$

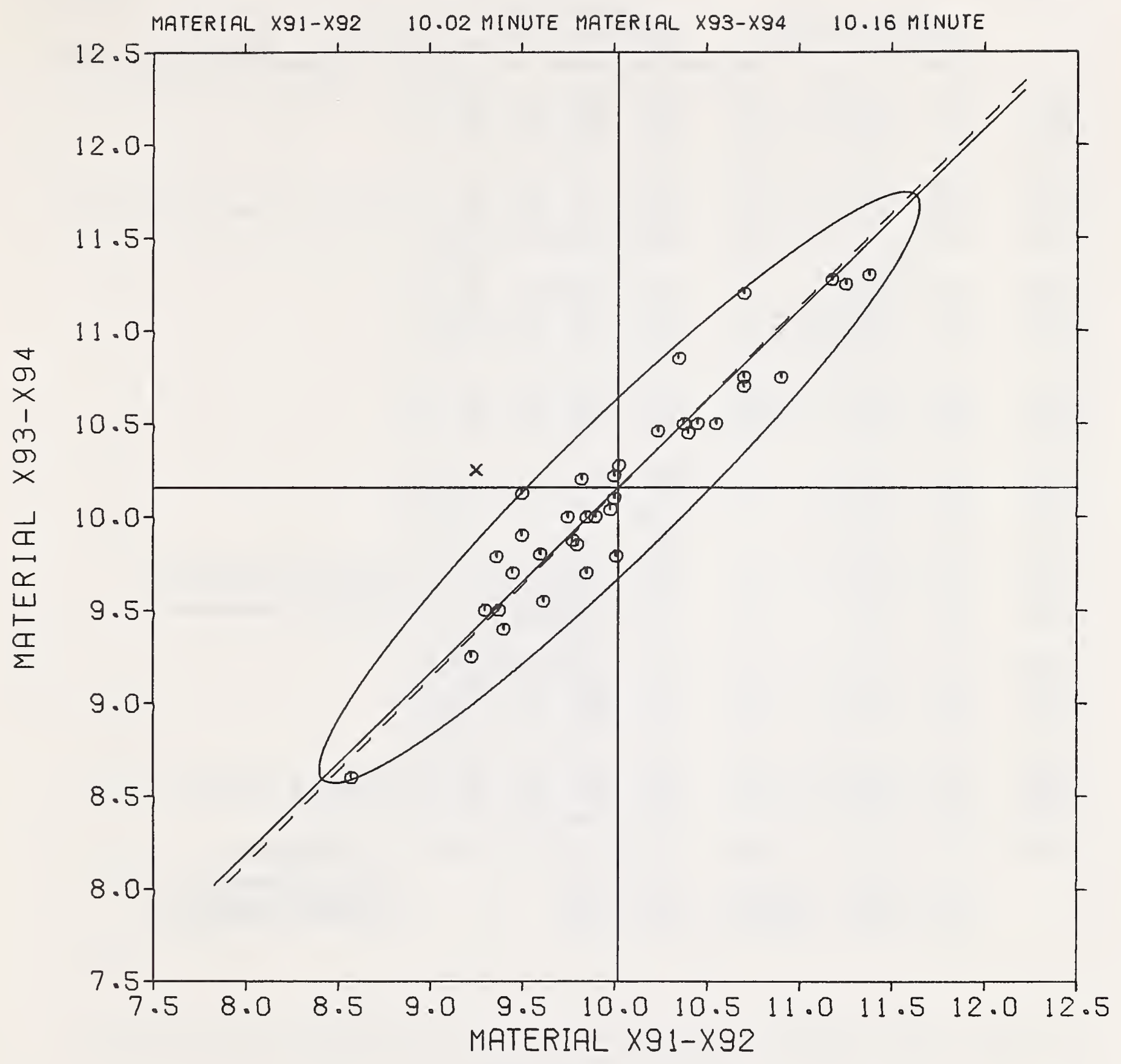




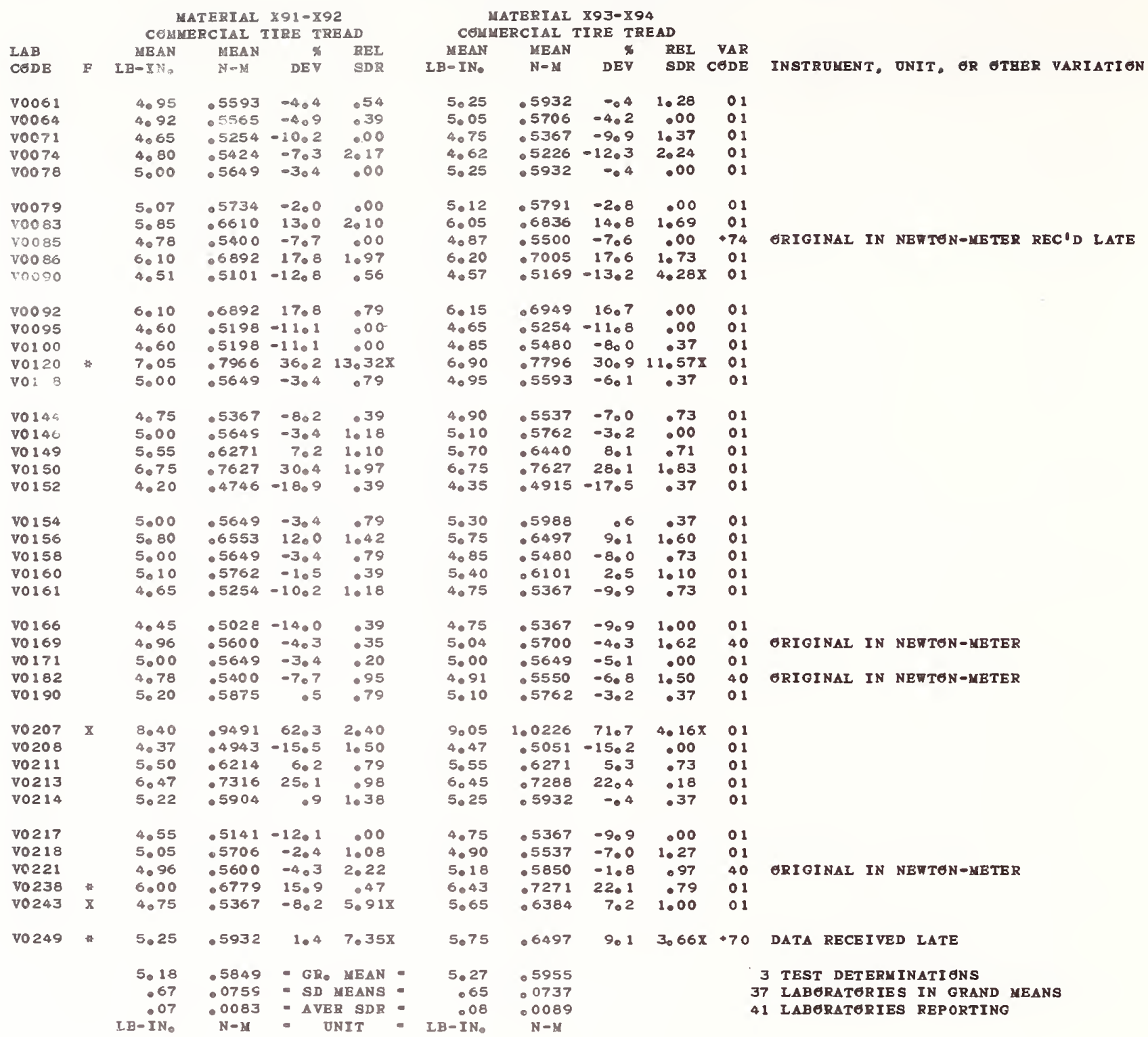


MINIMUM TORQUE

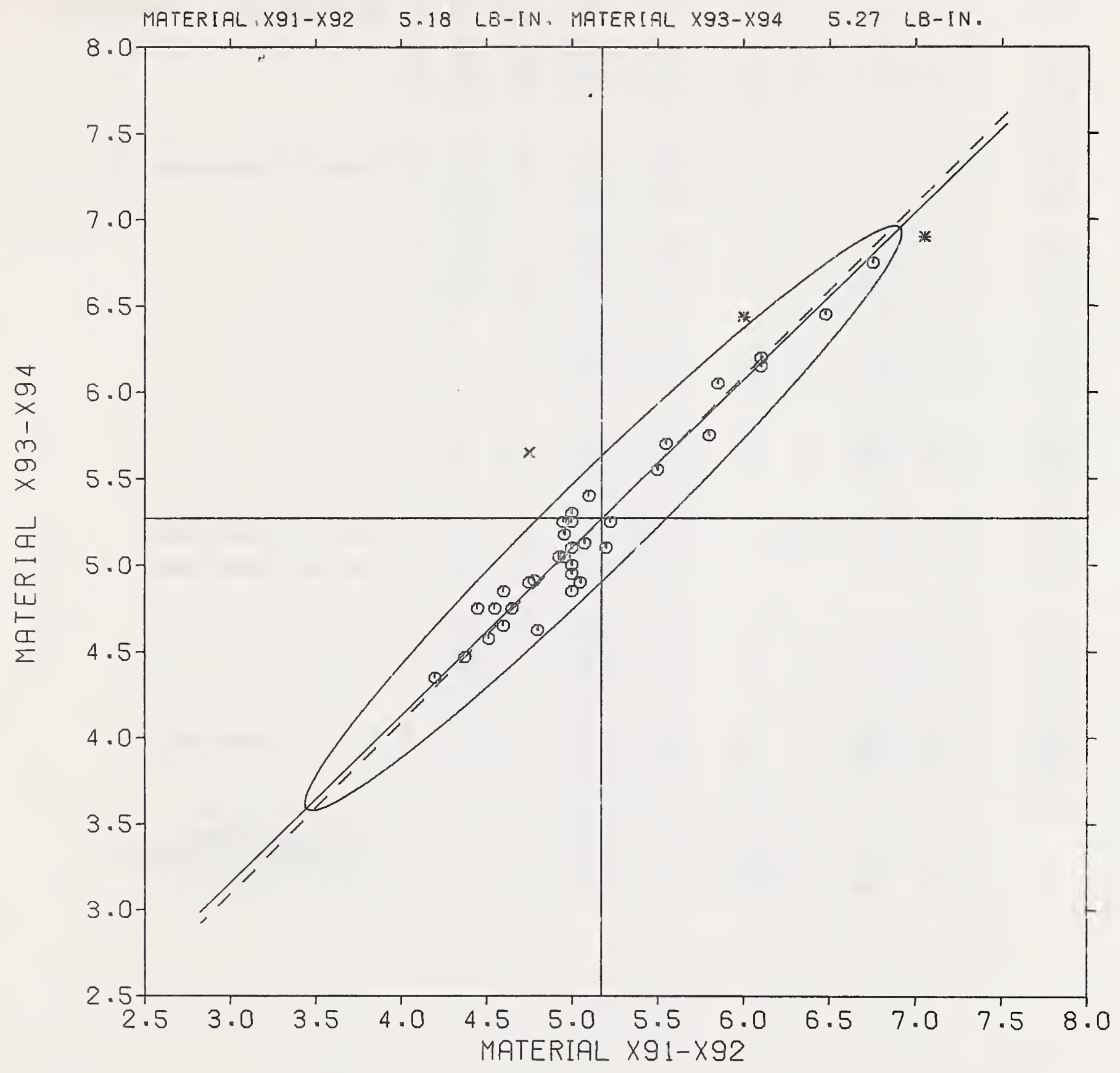




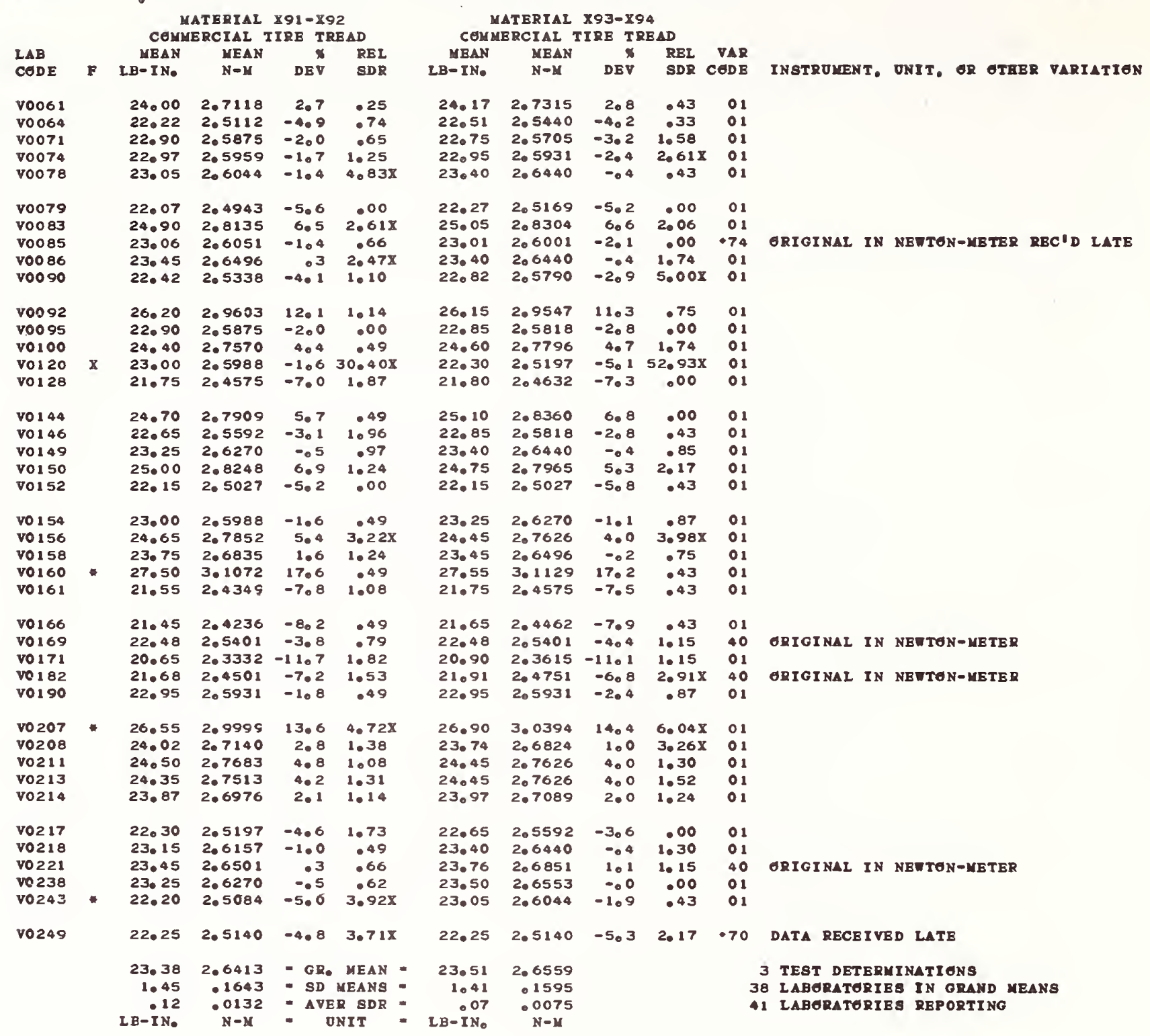


MAXIMUM TORQUE

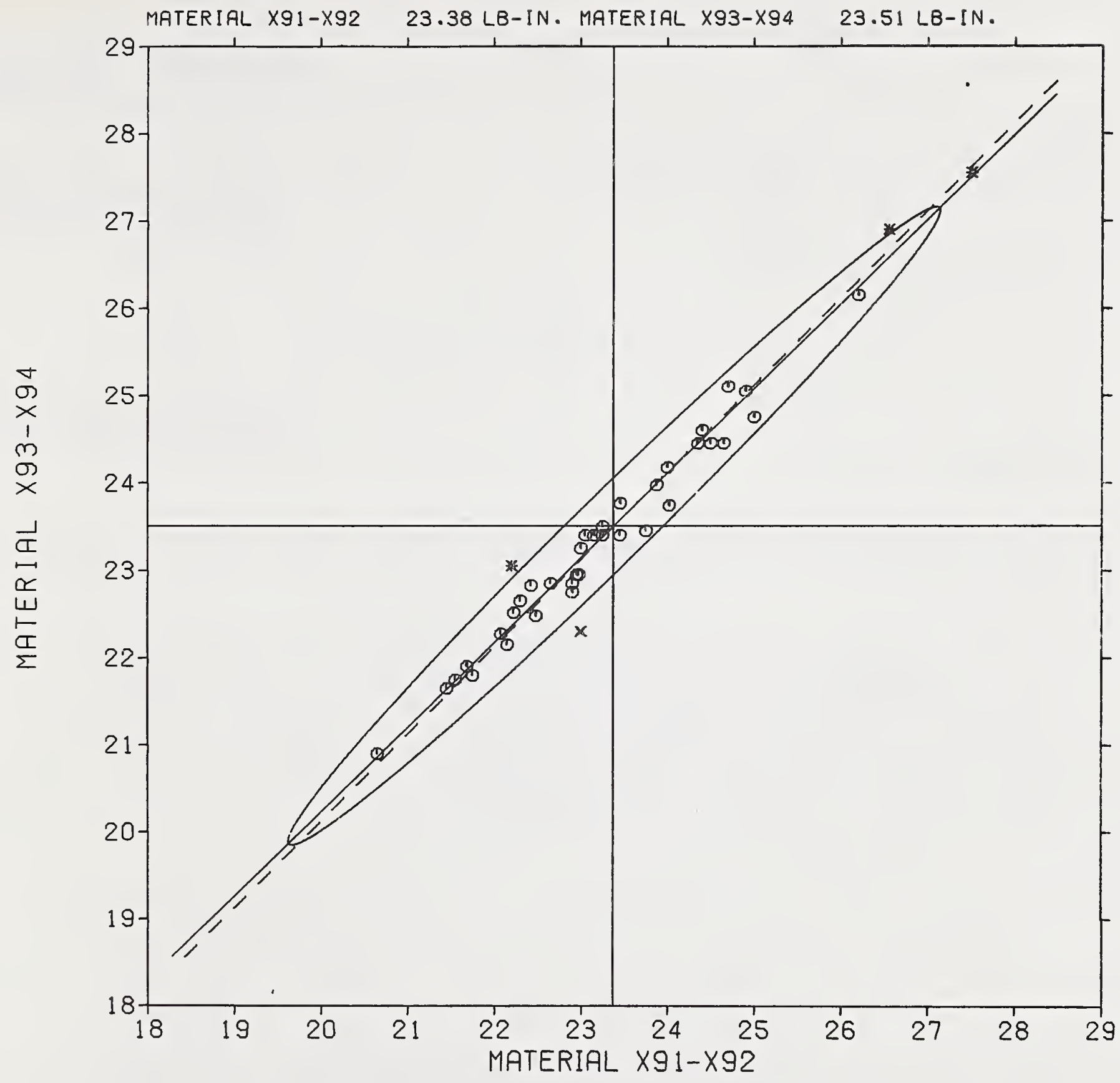


NBS-114A (REV. 9.78)

\begin{tabular}{|c|c|c|}
\hline $\begin{array}{l}\text { U.S. DEPT. OF COMM. } \\
\text { BIBLIOGRAPHIC DATA } \\
\text { SHEET }\end{array}$ & $\begin{array}{l}\text { 1. PUBLICATION OR REPORT NO. } \\
\text { RUBBER CRP } 40\end{array}$ & 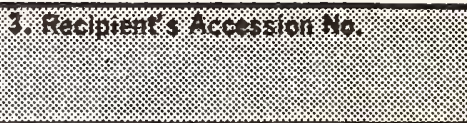 \\
\hline \multicolumn{2}{|l|}{ 4. TITLE AND SUBTITLE } & 5. Publication Date \\
\hline \multicolumn{2}{|c|}{ INTERLABORATORY PROGRAMS FOR RUBBER } & August 9, 1979 \\
\hline \multicolumn{2}{|l|}{ Analyses No. 40} & 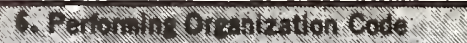 \\
\hline \multicolumn{2}{|l|}{ April - June 1979} & \\
\hline \multicolumn{2}{|l|}{ 7. AUTHOR(S) } & 8. Performing Organ. Report No. \\
\hline \multicolumn{2}{|c|}{ J. Horlick, G. W. Bullman, T. Cummings } & NBSIR 79-1805 \\
\hline \multicolumn{2}{|c|}{ 9. PERFORMING ORGANIZATION NAME AND ADDRESS } & 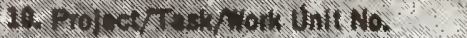 \\
\hline \multirow{2}{*}{\multicolumn{2}{|c|}{$\begin{array}{l}\text { NATIONAL BUREAU OF STANDARDS } \\
\text { DEPARTMENT OF COMMERCE } \\
\text { WASHINGTON, DC } 20234\end{array}$}} & \\
\hline & & 11. Contract/Grant No. \\
\hline \multirow{3}{*}{\multicolumn{2}{|c|}{$\begin{array}{l}\text { 12. SPONSORING ORGANIZATION NAME AND COMPLETE ADDRESS (Street, City, stato, zIP) } \\
\text { Collaborative Testing Services, Inc., } 9241 \text { Wood Glade Drive, } \\
\text { Great Fal1s, VA } 22066\end{array}$}} & 13. Type of Report \& Period Covered \\
\hline & & FINAL \\
\hline & & 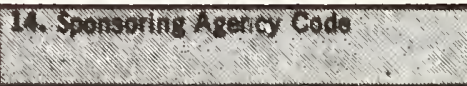 \\
\hline \multicolumn{3}{|l|}{ 15. SUPPLEMENTARY NOTES } \\
\hline
\end{tabular}

16. ABSTRACT (A 200-word or less factual summary of most sigrificant information. If document includes a significant bibliography or literature survey, mention it here.)

Collaborative Reference Programs provide participating laboratories with the means for checking periodically the level and uniformity of their testing in comparison with that of other participating laboratories. An important by-product of the programs is the provision of realistic pictures of the state of the testing art. This is one of the periodic reports showing averages for each participant, within and between laboratory variability, and other information for participants and standards committees.

17. KEY WORDS (six to twelve entries; alphebetical order; capitalize only the first letter of the first key word unless a proper namo; oeparated by semicolons)

Collaborative reference program; Laboratory evaluation; Precision; Reference samples; Rubber; Testing calibration.

XX For Official Distribution. Do flot Release to NTIS

Order From Sup. of Doc., U.S. Government Printing Office, Washington, DC 20402, SD Stock No. SN003-003-

Order From National Technical Information Service (NTIS), Springfield, VA. 2216

\begin{tabular}{|l|c|}
\hline $\begin{array}{l}\text { 19. SECURITY CLASS } \\
\text { (THIS REPORT) }\end{array}$ & $\begin{array}{c}\text { 21. NO. OF } \\
\text { PRINTED PAGES } \\
\text { UNCLASSIFIED }\end{array}$ \\
\hline $\begin{array}{l}\text { 20. SECURITY CLASS } \\
\text { (THIS PAGE) }\end{array}$ & 27 \\
UNCLASSIFIED & 22. Price \\
\hline
\end{tabular}



